\title{
Significance of assimilation and fractional crystallization (AFC) process in the generation of basaltic lava flows from Chhotaudepur area, Deccan Large Igneous Province, NW India
}

\author{
K R Hari ${ }^{1} *$, Vikas Swarnkar ${ }^{2}$ and M P Manu Prasanth ${ }^{1}$ \\ ${ }^{1}$ School of Studies in Geology and Water Resource Management, Pt. Ravishankar Shukla University, \\ Raipur 492 010, India. \\ ${ }^{2}$ Department of Civil Engineering, Yugantar Institute of Technology and Management, Rajnandgaon \\ 491441 , India. \\ *Corresponding author.e-mail: krharigeology@gmail.com
}

MS received 23 May 2017; revised 21 December 2017; accepted 22 December 2017; published online 2 August 2018

Basaltic and basaltic andesitic flows have been identified from the Chhotaudepur area in the Deccan Large Igneous Province. The $\mathrm{SiO}_{2}$ content of these flows varies from 46.7 to $53.7 \mathrm{wt} \%$. None of the samples have primary magma signatures as they exhibit low Mg\# (0.42-0.68), Ni (4.8-33.4 ppm) and $\mathrm{Cr}(33.08-143.06 \mathrm{ppm})$. Highly variable concentrations of the LILE [Rb $(2-74 \mathrm{ppm}), \mathrm{Ba}(52-351 \mathrm{ppm})$, Cs (0.1-1 ppm) and Sr (273-745 ppm)] and relatively enriched LREE are also noticed. The coherent chondrite normalized REE diagram and primitive mantle normalized multi-element diagram suggests a consanguinity among the flows. Low $(\mathrm{Nb} / \mathrm{Th})_{\mathrm{PM}}(0.30-1.09)$, high $(\mathrm{Th} / \mathrm{Yb})_{\mathrm{PM}}(3.09-16.58)$ ratios and marked variations in $\mathrm{Rb}$ concentration $(2.4-74.11 \mathrm{ppm})$ with variable $\mathrm{La} / \mathrm{Yb}(5.5-23.7)$ ratios suggests that magmas in the Chhotaudepur region were assimilated by the crustal components. The elevated $\mathrm{Th} / \mathrm{Ta}$ and $\mathrm{La} / \mathrm{Yb}$ relation further indicate concurrent assimilation and fractional crystallization process was involved in the genesis of the flows. Assimilation and fractional crystallization modelling of the flows was carried out with $20 \%$ olivine, $25 \%$ clinopyroxene, $45 \%$ plagioclase and $10 \%$ titano-magnetite as fractionating minerals and upper continental crust as the assimilant. The results reveal that all the flows were modified by AFC process.

Keywords. Deccan large igneous province; petrogenesis; concurrent assimilation and fractional crystallization.

\section{Introduction}

Flood basalt provinces represent important magmatic events which may contribute significantly to the generation of new continental crust. Origin of the continental flood basalt with eruptions of millions of cubic kilometers of basalt in a limited time span is still debatable (Gallagher and Hawkesworth 1992; Coffin and Eldholm 1994; Silver et al. 2006; Ernst and Bleeker 2010; Jowitt and Ernst 2013). The Deccan Large Igneous Province (DLIP) covering $\sim 500,000 \mathrm{~km}^{2}$ of Indian shield (figure 1) is mainly composed of nearly flat-lying tholeiitic flows (Bose 1972; Devey and Cox 1987; Hari et al. 2002). 


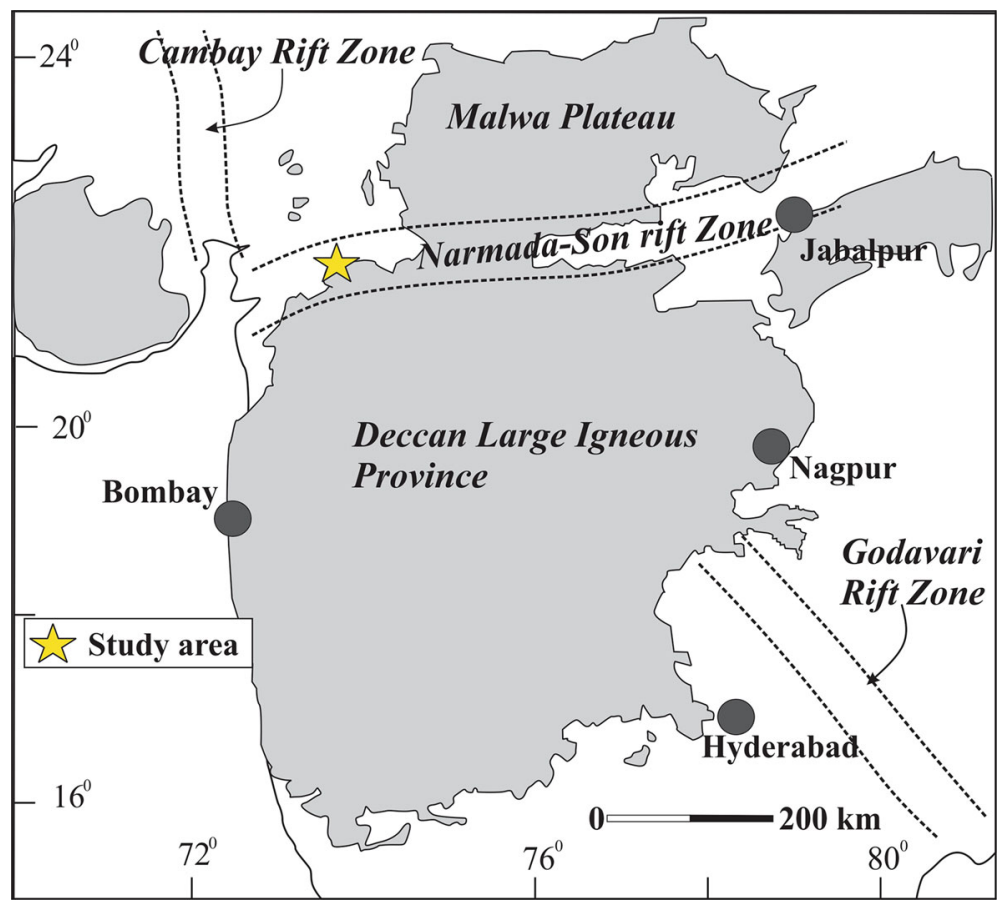

Figure 1. Map of Deccan Traps showing the location of the study area (after Krishnamurthy et al. 2000).

The original lava volume has been estimated as $(1-3) \times 10^{6} \mathrm{~km}^{3}$ (Sen 2001; Jay et al. 2009) with the majority of the eruption in a period less than 1 million year, with catastrophic consequences for life on Earth (Courtillot and Renne 2003; Chenet et al. 2007). Recently, on the basis of Chhattisgarh subsurface dykes and Mainpur orangeites, Rao et al. (2011) recalculated the areal extent of Deccan lavas as $8.5 \times 10^{4} \mathrm{~km}^{2}$. Deccan volcanic event attributed to the impingement of the Réunion mantle plume and culminated in the final phase of rupture of the Gondwana supercontinent (Mahoney et al. 2002; Collier et al. 2008).

The DLIP is characterized by both tholeiitic and alkali basalts as well as relatively minor alkaline and carbonatitic intrusions (Basu et al. 1993; Rock et al. 1994; Simonetti et al. 1998; Shrivastava and Pattanayak 2002; Ray et al. 2007; Sen et al. 2009). Alkaline rocks of DLIP is dominated by silica undersaturated rocks such as nephelinites, basanite and tephrites, whereas the alkaline silicasaturated rocks, such as trachytes and syenites, form a minor component (Devey and Cox 1987; Peng et al. 1994; Rock et al. 1994; Greenough et al. 1998; Ray et al. 2007).

During the ascent of a plume-related magma through the continental crust, various magma modification processes such as fractional crystallization and crustal contamination will take place, which eventually changes the composition of the original magma. The concurrent assimilation and fractional crystallization process is regarded as one of the important processes which change the primary nature of the ascending magma (DePaolo 1981; Lightfoot and Hawkesworth 1988; Dessai et al. 2008). In the present paper, we tried to discuss the role of assimilation and fractional crystallization (AFC) on the magma modification process of the flows from the Chhotaudepur area of DLIP.

\section{General geology}

The Chhotaudepur area, which is located in the northern parts of DLIP (figure 2), comprises mainly of two igneous complexes, the Ambadungar alkaline igneous complex, located in the southeastern corner of DLIP and the Phenai Mata igneous complex, which is located in the western part (Randive et al. 2017). Many workers (e.g., Basu et al. 1993; Rock et al. 1994; Greenough et al. 1998; Hari 1998; Hari et al. 2000, 2014; Shrivastava and Pattanayak 2002; Ravi Kumar and Mohan 2005; Sen et al. 2009; Randive 2015) reported dyke swarms of basalt, dolerite, lamprophyre and tinguite from the Chhotaudepur area. The Ambadungar complex of Chhotaudepur area is dominated by calcitecarbonatites associated with a small portion of redbrown ankeritic-carbonatite. Phenai Mata igneous complex is dominantly composed of tholeiitic 
$73^{\circ} 50^{\prime}$

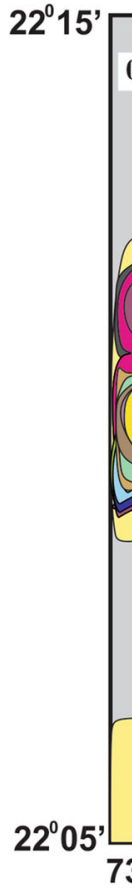

\section{0}

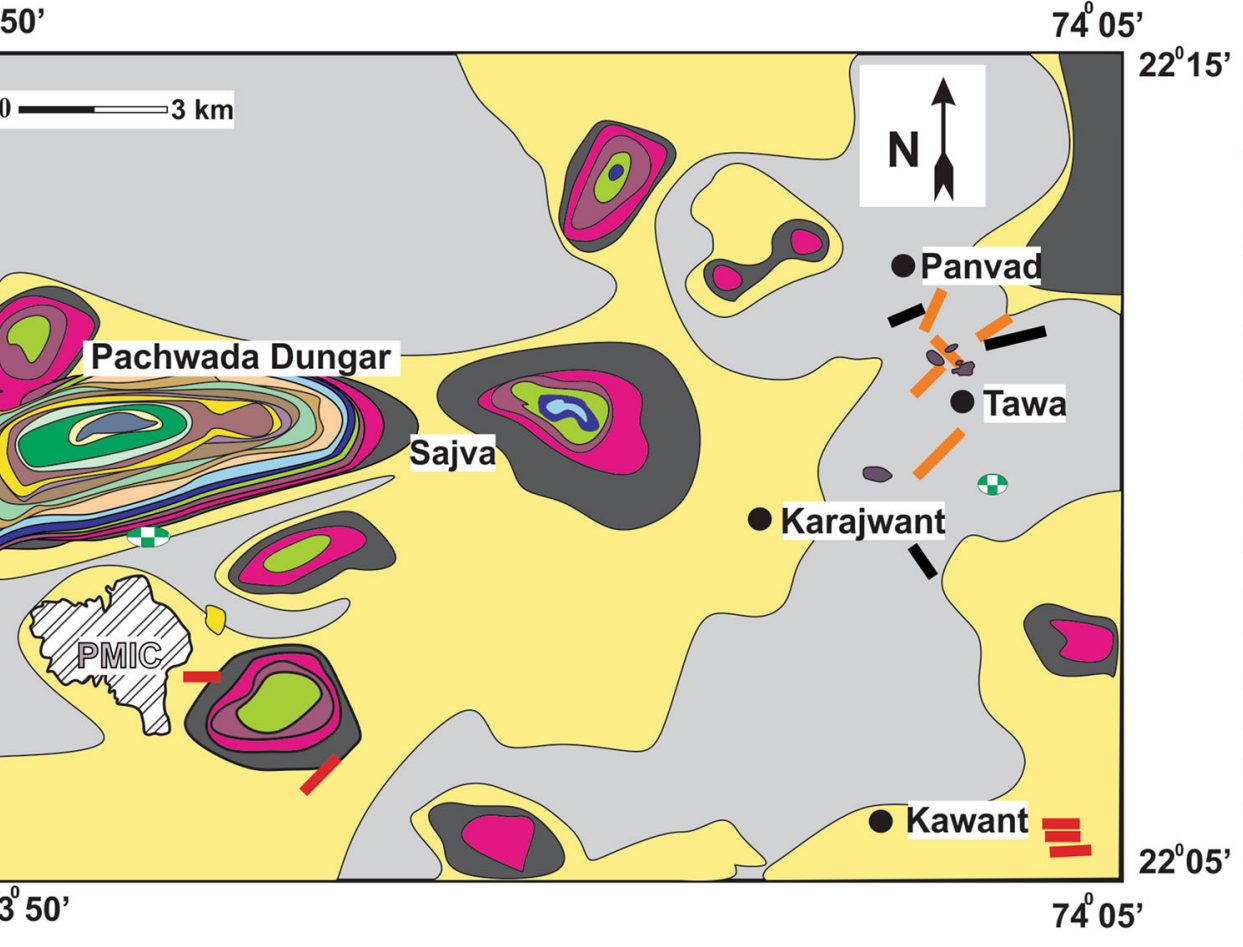

$74^{\circ} 05^{\prime}$

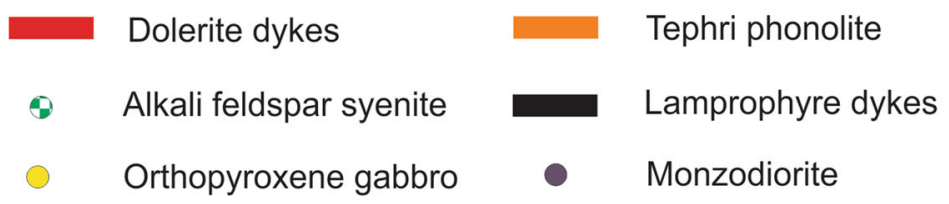

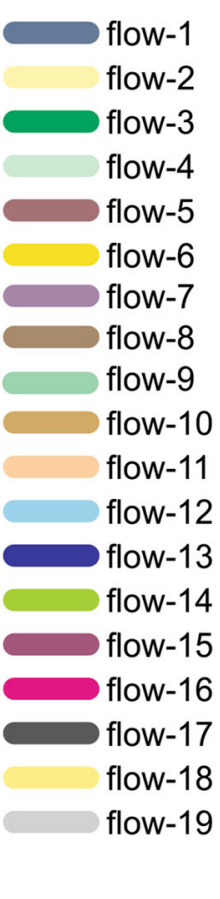

Figure 2. Flow map of the Chhotaudepur region (modified after Hari et al. 2014). PMIC - Phenai Mata Igneous Complex.

basalt, felsic rocks, gabbro and orthopyroxene gabbro (Hari et al. 2000). Hari et al. (2014) reported alkali feldspar syenite with shoshonitic affinity from the Phenai Mata region and proposed a metasomatic genesis for these rocks. Excluding Phenai Mata igneous complex and Ambadungar alkaline complex, the entire area is composed of basalt and basaltic andesite flows. For flow demarcation the methods used by Ganguly et al. (2012, 2014) are taken into consideration and based on field and petrographic studies, nineteen flows (figure 2) were identified in the present area. Most of the flows are characterized by amygdules, spheroidal weathering, red boles and columnar joints. The top flows (mainly second, sixth, tenth and thirteenth flows) are characterized by vesicularity along with the few amygdules. Feeble columnar structures were noticed in the third, fifth and seventh flows. Eighth and ninth, eleventh and twelfth, fourteenth and fifteenth flows can be demarcated by the presence of red boles between them. Further, for more accurate flow demarcation of the entire sequence, petrographic studies were also taken into consideration.

\section{Petrography}

Some of the flows have olivine, pyroxene and plagioclase as phenocrysts and the ground mass is generally comprised of plagioclase, clinopyroxene, glass and opaques (figure 3). Alteration along the boundaries and fracture zones of olivine grains are observed in most of the thin sections (figure 3a). The glomeroporphyritic texture is noticeable in some sections (figure $3 \mathrm{~b}$ ). In few cases, complete alteration of olivine grains either to serpentine or to iddingsite are also perceptible. Clinopyroxenes are identified as augites and the plagioclase grains are identified as labradorites. Few flows have plagioclase and pyroxene phenocrysts set in a matrix mainly composed of plagioclase laths with clinopyroxene, glass, and opaques. In few flows, sporadic plagioclase phenocrysts are noticeable (figure $3 \mathrm{c}$ ). 

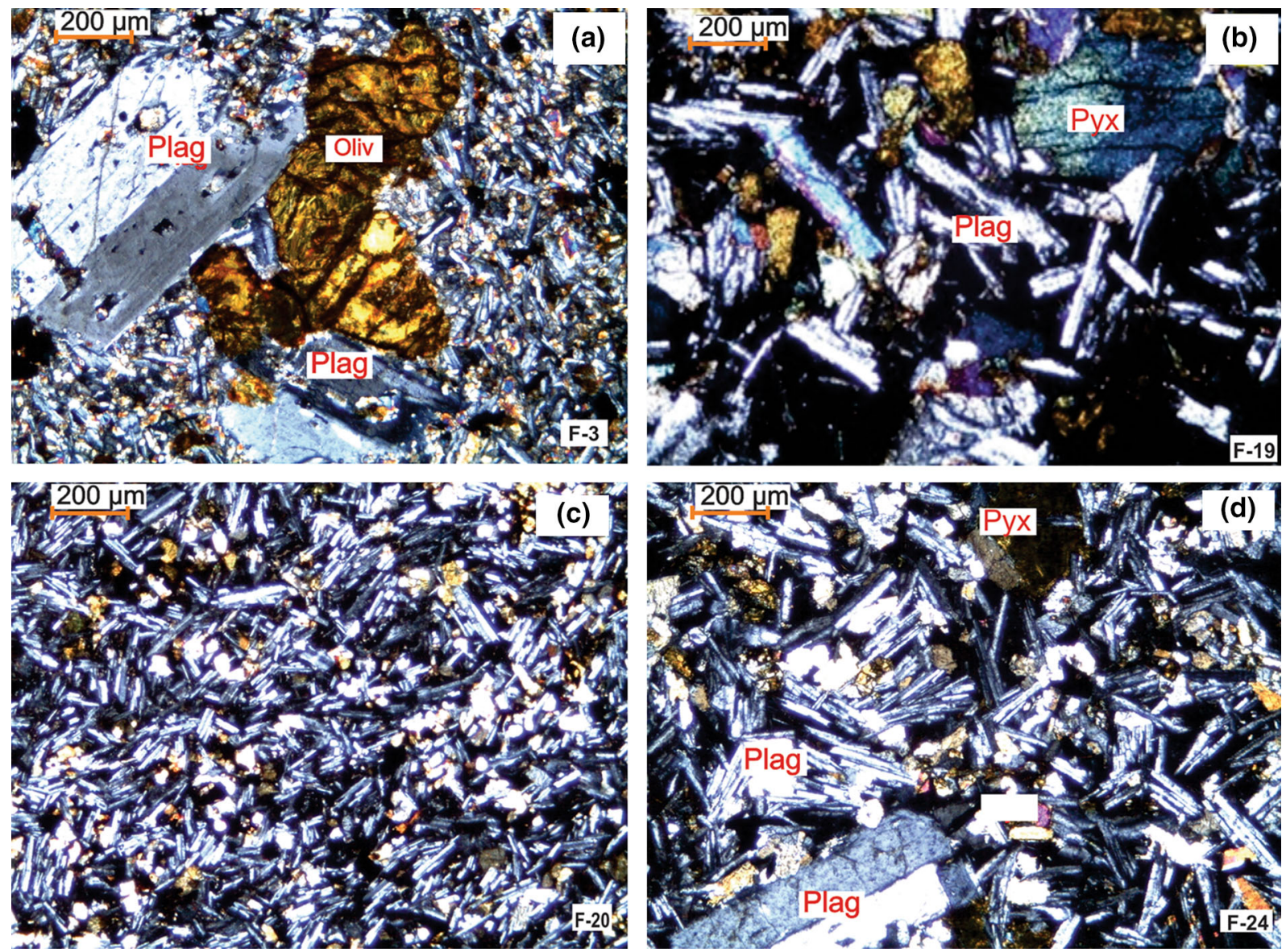

Figure 3. Photomicrographs showing (a) clustered plagioclase phenocrysts, altered olivine and pyroxene forming glomeroporphyritic texture in a ground mass comprised of plagioclase, clinopyroxenes, and opaques. Zoning is observed in plagioclase phenocrysts, (b) altered olivine, pyroxene and plagioclase phenocrysts set in the matrix of plagioclase, clinopyroxenes, and opaques, (c) plagioclase and pyroxene grains in a non-porphyritic flow, and (d) plagioclase phenocrysts in the groundmass of plagioclase, clinopyroxene, and opaques exhibiting intergranular and intersertal texture.

It is noticeable that all these flows have parallel arrangement of plagioclase laths and exhibiting characteristic flow texture in the groundmass. Higher concentration of opaques with few plagioclase phenocrysts are noticeable in few flows. Some flows exhibit intersertel and intergranular textural patterns also (figure 3c and d).

\section{Analytical techniques}

Least altered samples of basalts and basaltic andesites were collected from the Chhotaudepur area and analyzed for major, trace and rare earth elemental compositions. Samples were pulverized in an agate mortar and pestle. Major elements were analyzed by XRF-Phillips MAGIX PRO (Model 2440) using pressed powder pellets, with relative standard deviations $<3 \%$ and totals were all $100 \pm 1 \%$. Rare earth elements, HFSEs and other trace elements were determined by ICPMS (Perkin Elmer SCIEX ELAN DRC II) at the National Geophysical Research Institute (NGRI), Hyderabad. A mixture of double distilled acid $\left(\mathrm{HF}+\mathrm{HNO}_{3}+\mathrm{HCl}, 5: 3: 2 \mathrm{ml}\right)$ was added to $0.05 \mathrm{~g}$ of rock powder in Savillex vessels and kept on a hot plate at $150^{\circ} \mathrm{C}$ for 3 days. After 3 days, the entire mixture was evaporated to dryness. The decomposition procedure was repeated by adding $5 \mathrm{ml}$ of the acid mixture $\left(\mathrm{HF}+\mathrm{HNO}_{3}\right.$ $+\mathrm{HCl}, 5: 3: 2 \mathrm{ml}$ ) for two days. After that, 20 $\mathrm{ml}$ of 1:1 $\mathrm{HNO}_{3}$ was added to the dried samples and heated at $150^{\circ} \mathrm{C}$ for $10-15 \mathrm{~min}$. Then $5 \mathrm{ml} \mathrm{Rh} \mathrm{(1} \mathrm{ppm)} \mathrm{was} \mathrm{added} \mathrm{as} \mathrm{the} \mathrm{internal}$ standard. After cooling, the volume of the prepared solution is made up to $250 \mathrm{ml}$. Certified reference materials JB-2 and JGb- 1 were run as standard martials along with the samples. 
Precision and reproducibility obtained for international reference materials are given in table 1 . The chondrite normalized and primitive mantle normalized ratios were calculated from the values of Sun and McDonough (1989). The result of the analyses are given in table 2 .

\section{Geochemistry}

In the total alkali $\left(\mathrm{Na}_{2} \mathrm{O}+\mathrm{K}_{2} \mathrm{O}\right)$ vs. silica $\left(\mathrm{SiO}_{2}\right)$, diagram the samples plot in the fields of basalt and basaltic andesite (figure 4). The $\mathrm{SiO}_{2}$ wt\% of the basalts and basaltic andesites ranges from 46.7 to 53.7. The $\mathrm{Al}_{2} \mathrm{O}_{3}$ content shows a narrow range between 11.5 and $13.2 \mathrm{wt} \%$. Moderate to high variations in $\mathrm{MgO}$ and $\mathrm{CaO}$ (6.3-9.9 and 6.5$11.75 \mathrm{wt} \%$, respectively) content were also noticed. The $\mathrm{Mg} \#$ values range from 0.42 to 0.68 . Based on the $\mathrm{TiO}_{2}$ (1.94-3.4 wt\%) content, these rocks are classified as high Ti-basalts $\left(\mathrm{TiO}_{2} \geq 2 \mathrm{wt} \%\right)$. CIPW normative compositions are marked by the presence of quartz (2.1-8.2 wt\%), albite (17.0-21.1 wt\%) anorthite (19.4-22.2 wt\%), diopside (4.5$22.1 \mathrm{wt} \%)$ and hypersthene (9.5-16.0 wt\%).

All the samples have slightly higher concentrations of large ion lithophile elements (LILE) and light rare earth elements (LREE) (table 2). Low concentration of $\mathrm{Ni}(4.8-33.4 \mathrm{ppm})$ and $\mathrm{Cr}$ (33.08$143.06 \mathrm{ppm})$ relative to the primary mantle melts $(\mathrm{Mg} \# \sim 0.7, \mathrm{Ni}>400-500 \mathrm{ppm}, \mathrm{Cr}>1000 \mathrm{ppm})$ (Wilson 1989) are observed in these rocks. The highly variable $\mathrm{Rb}(2-74 \mathrm{ppm})$ and $\mathrm{Ba}$ (52.09$1231.2 \mathrm{ppm}$ ) concentrations in the studied samples is likely to be caused by post-crystallization alteration processes or crustal contamination. Higher Sr (273.1-745.9 ppm) content and Rb (2-74 ppm) contents compared to OIB (Rb: 31 and Sr: 660 ppm) (Sun and McDonough 1989) are noticeable in these rocks. In comparison with the $\mathrm{Nb}$ and Ta concentrations of OIB (Nb: 48 and Ta: 2.7 ppm) (Sun and McDonough 1989; Iwamori and Nakamura 2015), the $\mathrm{Nb}(10.40-26.3 \mathrm{ppm})$ and $\mathrm{Ta}(0.72-1.8 \mathrm{ppm})$ contents of the studied samples exhibit relatively lower values, except for one sample (F-36). Even though, some of the basaltic flows in the present area exhibit slightly higher $\mathrm{Mg} \#$ values (0.68), their low $\mathrm{Ni}$ and $\mathrm{Cr}$ concentrations (table 2) negate their primary magma signature suggesting that magma modification might have taken place.

Many researchers suggested the fractional crystallization as an important differentiation process which operated during the evolution of basaltic lava flows of Deccan Traps, particularly in the lava flows of Indore-Khargaon, Mhow-Chikaldara (Chatterjee and Bhattacharji 2001), Jabalpur and Seoni areas (Shrivastava and Pattanayak 2002; Kumar and Shrivastava 2009), Western Ghats section (Mahoney et al. 2002; Melluso et al. 2004, 2006) and Bijasan Ghat section of Satpura Range (Sheth et al. 2004). In order to assess the fractional crystallization process, $\mathrm{Ni}, \mathrm{Cr}$, and $\mathrm{CaO}$ were plotted against $\mathrm{MgO}$. Positive correlation of $\mathrm{Ni}$ and $\mathrm{Cr}$ with $\mathrm{MgO}$ suggests olivine as well as clinopyroxene fractionation (figure 5), whereas the positive correlation of $\mathrm{CaO}$ with $\mathrm{MgO}$ suggests plagioclase fractionation. Slight Eu anomaly of some samples in the chondrite-normalized REE diagram (figure 6a) also substantiates plagioclase fractionation.

The chondrite normalized REE (Sun and McDonough 1989) patterns (figure 6a) exhibit enrichment in LREE relative to the HREE. Minor $\mathrm{Eu}$ anomalies and fractionated HREE patterns are also noticeable. The coherent REE chondrite normalized patterns suggest similar genetic relationships among the flows. The primitive mantle normalized (Sun and McDonough 1989) multielement patterns of the samples also exhibits overall geochemical coherence among the flows from the Chhotaudepur area (figure 6b). Distinct negative $\mathrm{Zr}$ anomalies are noticeable in all the samples. Positive Th, Ta, La-Ce, Gd-Tb anomalies and negative $\mathrm{U}, \mathrm{Ti}$ and $\mathrm{Yb}$ anomalies are also noticeable in the primitive mantle normalized multi-element diagram.

\section{Discussion}

\subsection{Assimilation and fractional crystallization of Deccan magmas}

Assimilation of crustal material in mantle-derived magmas during their ascent to the surface through continental crust has been considered as an important process for the compositional diversity (Hawkesworth et al. 1984; Rudnick et al. 1986; Nicholson et al. 1991; Grunder 1992; Cribb and Barton 1996; Beard et al. 2005; Tegner et al. 2005; Michael and Cheadle 2009; Freund et al. 2013; Genske et al. 2013; Azzone et al. 2016). Ray (1998) on the basis of the $\mathrm{Sr}$ isotope systematics, concluded that alkaline silicate rocks of DLIP could be formed due to the crustal contamination 
Table 1. Major and trace element concentrations of JB-2 and JGb-1 obtained from XRF and HR-ICP-MS.

\begin{tabular}{|c|c|c|c|c|c|c|c|c|}
\hline \multirow[b]{2}{*}{$\mathrm{wt} \%$} & \multicolumn{4}{|c|}{ JB-2 } & \multicolumn{4}{|c|}{ JGb-1 } \\
\hline & A & B & $\mathrm{SD}$ & $\mathrm{RSD}(\%)$ & A & B & $\mathrm{SD}$ & $\mathrm{RSD}(\%)$ \\
\hline $\mathrm{SiO}_{2}$ & 53.180 & 53.1 & 0.020 & 0.038 & 43.380 & 43.4 & 0.042 & 0.098 \\
\hline $\mathrm{Al}_{2} \mathrm{O}_{3}$ & 14.680 & 14.620 & 0.030 & 0.204 & 17.600 & 17.660 & 0.042 & 0.241 \\
\hline $\mathrm{Fe}_{2} \mathrm{O}_{3}$ & 13.980 & 14.100 & 0.060 & 0.429 & 15.330 & 15.330 & 0.000 & 0.000 \\
\hline $\mathrm{MnO}$ & 0.210 & 0.210 & 0.000 & 0.000 & 0.170 & 0.170 & 0.000 & 0.000 \\
\hline $\mathrm{MgO}$ & 4.420 & 4.430 & 0.005 & 0.113 & 7.940 & 7.950 & 0.007 & 0.089 \\
\hline $\mathrm{CaO}$ & 9.790 & 9.852 & 0.031 & 0.317 & 11.900 & 11.980 & 0.057 & 0.475 \\
\hline $\mathrm{Na}_{2} \mathrm{O}$ & 2.080 & 2.054 & 0.013 & 0.625 & 1.200 & 1.230 & 0.021 & 1.768 \\
\hline $\mathrm{K}_{2} \mathrm{O}$ & 0.430 & 0.422 & 0.004 & 0.884 & 0.246 & 0.240 & 0.004 & 1.725 \\
\hline $\mathrm{TiO}_{2}$ & 1.158 & 1.167 & 0.005 & 0.389 & 1.700 & 1.720 & 0.014 & 0.832 \\
\hline $\begin{array}{l}\mathrm{P}_{2} \mathrm{O}_{5} \\
\text { ppm }\end{array}$ & 0.100 & 0.100 & 0.000 & 0.000 & 0.050 & 0.050 & 0.000 & 0.000 \\
\hline $\mathrm{Sc}$ & 54.268 & 54.400 & 0.066 & 0.122 & 37.235 & 36.600 & 0.449 & 1.205 \\
\hline V & 588.010 & 578.0 & 5.005 & 0.851 & 645.4 & 640.0 & 3.825 & 0.593 \\
\hline $\mathrm{Cr}$ & 26.760 & 27.4 & 0.320 & 1.196 & 59.8 & 59.3 & 0.343 & 0.574 \\
\hline $\mathrm{Co}$ & 39.015 & 39.8 & 0.392 & 1.006 & 61.6 & 61.6 & 0.010 & 0.016 \\
\hline $\mathrm{Ni}$ & 14.068 & 14.2 & 0.066 & 0.469 & 25.7 & 25.4 & 0.195 & 0.759 \\
\hline $\mathrm{Cu}$ & 222.346 & 227.0 & 2.327 & 1.047 & 86.8 & 86.8 & 0.016 & 0.019 \\
\hline $\mathrm{Zn}$ & 109.780 & 110.0 & 0.110 & 0.100 & 109.3 & 111.0 & 1.187 & 1.086 \\
\hline $\mathrm{Ga}$ & 16.381 & 17.0 & 0.310 & 1.891 & 18.9 & 18.9 & 0.031 & 0.165 \\
\hline $\mathrm{Rb}$ & 6.155 & 6.2 & 0.023 & 0.366 & 4.0 & 4.0 & 0.011 & 0.264 \\
\hline $\mathrm{Sr}$ & 180.273 & 178.0 & 1.137 & 0.630 & 320.6 & 321.0 & 0.293 & 0.091 \\
\hline $\mathrm{Y}$ & 25.086 & 24.9 & 0.093 & 0.370 & 10.8 & 10.8 & 0.068 & 0.623 \\
\hline $\mathrm{Zr}$ & 51.560 & 51.4 & 0.080 & 0.155 & 33.5 & 33.5 & 0.033 & 0.099 \\
\hline $\mathrm{Nb}$ & 0.943 & 0.8 & 0.072 & 7.582 & 2.8 & 2.8 & 0.005 & 0.177 \\
\hline Cs & 0.923 & 0.9 & 0.012 & 1.246 & 0.3 & 0.3 & 0.007 & 2.579 \\
\hline $\mathrm{Ba}$ & 215.951 & 208.0 & 3.976 & 1.841 & 63.0 & 63.0 & 0.015 & 0.024 \\
\hline $\mathrm{La}$ & 2.479 & 2.4 & 0.055 & 2.198 & 3.8 & 3.7 & 0.053 & 1.399 \\
\hline $\mathrm{Ce}$ & 7.044 & 6.8 & 0.137 & 1.942 & 7.9 & 7.9 & 0.038 & 0.482 \\
\hline $\operatorname{Pr}$ & 1.004 & 1.0 & 0.022 & 2.167 & 1.2 & 1.1 & 0.011 & 0.979 \\
\hline $\mathrm{Nd}$ & 6.940 & 6.7 & 0.120 & 1.729 & 5.7 & 5.7 & 0.004 & 0.069 \\
\hline $\mathrm{Sm}$ & 2.426 & 2.3 & 0.088 & 3.627 & 1.4 & 1.5 & 0.045 & 3.174 \\
\hline $\mathrm{Eu}$ & 0.917 & 0.9 & 0.029 & 3.108 & 0.6 & 0.6 & 0.001 & 0.225 \\
\hline $\mathrm{Gd}$ & 3.470 & 3.3 & 0.095 & 2.738 & 1.6 & 1.6 & 0.003 & 0.196 \\
\hline $\mathrm{Tb}$ & 0.675 & 0.6 & 0.028 & 4.074 & 0.3 & 0.3 & 0.012 & 3.573 \\
\hline Dy & 3.816 & 3.7 & 0.078 & 2.038 & 1.5 & 1.5 & 0.001 & 0.046 \\
\hline Но & 0.886 & 0.8 & 0.038 & 4.289 & 0.3 & 0.3 & 0.005 & 1.408 \\
\hline Er & 2.927 & 2.6 & 0.149 & 5.073 & 1.1 & 1.1 & 0.036 & 3.247 \\
\hline $\mathrm{Tm}$ & 0.503 & 0.5 & 0.027 & 5.268 & 0.2 & 0.2 & 0.010 & 6.036 \\
\hline $\mathrm{Yb}$ & 2.681 & 2.5 & 0.085 & 3.180 & 1.0 & 1.0 & 0.003 & 0.327 \\
\hline $\mathrm{Lu}$ & 0.434 & 0.4 & 0.022 & 5.017 & 0.1 & 0.2 & 0.001 & 0.956 \\
\hline Hf & 1.575 & 1.4 & 0.077 & 4.906 & 0.9 & 0.9 & 0.029 & 3.148 \\
\hline $\mathrm{Ta}$ & 0.243 & 0.2 & 0.022 & 8.848 & 0.2 & 0.2 & 0.007 & 4.113 \\
\hline $\mathrm{Pb}$ & 6.052 & 5.4 & 0.326 & 5.383 & 2.0 & 1.9 & 0.063 & 3.147 \\
\hline $\mathrm{Th}$ & 0.368 & 0.3 & 0.019 & 5.102 & 0.6 & 0.5 & 0.037 & 6.318 \\
\hline $\mathrm{U}$ & 0.184 & 0.2 & 0.012 & 6.522 & 0.2 & 0.2 & 0.004 & 2.501 \\
\hline
\end{tabular}

A: Present study (average of three values); B: reported values from Govindaraju GEOREM (georem.mpch-mainz.gwdg.de). SD: Standard deviation, RSD: relative standard deviation calculated w.r.t. the values given in column A. 
Table 2. Whole rock geochemical data for the basaltic lava flows from Chhotaudepur area, DLIP.

\begin{tabular}{|c|c|c|c|c|c|c|c|c|c|}
\hline $\begin{array}{l}\text { Flow no. } \\
\text { Sample }\end{array}$ & $\begin{array}{c}1 \\
F-2\end{array}$ & $\begin{array}{c}2 \\
\mathrm{~F}-3\end{array}$ & $\begin{array}{c}3 \\
\text { F-8 }\end{array}$ & $\begin{array}{c}4 \\
\mathrm{~F}-12\end{array}$ & $\begin{array}{c}5 \\
\text { F-13B }\end{array}$ & $\begin{array}{c}6 \\
\mathrm{~F}-14\end{array}$ & $\begin{array}{c}7 \\
\mathrm{~F}-16\end{array}$ & $\begin{array}{c}8 \\
\text { F-18 }\end{array}$ & $\begin{array}{c}9 \\
\text { F-19 }\end{array}$ \\
\hline $\mathrm{SiO}_{2}$ & 48.1 & 48.2 & 46.9 & 46.7 & 49.5 & 47.8 & 47.17 & 48.5 & 48.7 \\
\hline $\mathrm{Al}_{2} \mathrm{O}_{3}$ & 12.9 & 13.01 & 11.83 & 11.5 & 12.2 & 12.21 & 12.72 & 12.89 & 12.47 \\
\hline $\mathrm{Fe}_{2} \mathrm{O}_{3}$ & 13.64 & 13.87 & 13.45 & 15.74 & 10.35 & 14.57 & 13.53 & 14.99 & 13.91 \\
\hline $\mathrm{MnO}$ & 0.12 & 0.13 & 0.13 & 0.17 & 0.11 & 0.16 & 0.12 & 0.1 & 0.14 \\
\hline $\mathrm{MgO}$ & 7.11 & 7.21 & 9.39 & 8.65 & 9.91 & 6.43 & 8.72 & 6.35 & 7.08 \\
\hline $\mathrm{CaO}$ & 10.66 & 10.51 & 11.75 & 9.64 & 11.46 & 10.3 & 10.2 & 8.46 & 9.18 \\
\hline $\mathrm{Na}_{2} \mathrm{O}$ & 2.46 & 2.5 & 2.01 & 2.24 & 2.09 & 2.45 & 2.38 & 2.43 & 2.19 \\
\hline $\mathrm{K}_{2} \mathrm{O}$ & 0.65 & 0.69 & 0.57 & 0.65 & 0.7 & 0.75 & 0.84 & 0.71 & 0.94 \\
\hline $\mathrm{TiO}_{2}$ & 2.46 & 2.48 & 2.17 & 3.37 & 1.94 & 3.25 & 2.65 & 3.4 & 3.12 \\
\hline $\mathrm{P}_{2} \mathrm{O}_{5}$ & 0.24 & 0.26 & 0.18 & 0.29 & 0.24 & 0.36 & 0.42 & 0.39 & 0.73 \\
\hline $\mathrm{Sc}$ & 36.244 & 38.99 & 38.011 & 29.927 & 34.34 & 32.697 & 32.944 & 35.853 & 27.959 \\
\hline $\mathrm{V}$ & 394.985 & 421.465 & 370.235 & 436.976 & 288.708 & 460.618 & 361.138 & 506.155 & 337.988 \\
\hline $\mathrm{Cr}$ & 105.237 & 95.398 & 101.641 & 57.879 & 143.06 & 49.593 & 66.27 & 62.346 & 60.341 \\
\hline $\mathrm{Co}$ & 51.2 & 54.112 & 54.201 & 49.683 & 44.58 & 53.513 & 48.275 & 51.928 & 42.192 \\
\hline $\mathrm{Ni}$ & 33.407 & 32.696 & 29.856 & 16.249 & 32.625 & 16.913 & 22.964 & 24.18 & 11.54 \\
\hline $\mathrm{Cu}$ & 157.261 & 220.56 & 140.328 & 189.491 & 114.634 & 188.208 & 139.565 & 227.345 & 55.64 \\
\hline $\mathrm{Zn}$ & 86.066 & 89.091 & 76.459 & 95.343 & 69.455 & 103.284 & 98.914 & 131.228 & 95.803 \\
\hline $\mathrm{Ga}$ & 21.476 & 22.157 & 20.063 & 21.559 & 19.005 & 23.152 & 22.561 & 24.87 & 20.194 \\
\hline $\mathrm{Rb}$ & 4.054 & 8.386 & 2.45 & 5.341 & 6.894 & 8.474 & 20.474 & 9.212 & 18.346 \\
\hline $\mathrm{Sr}$ & 273.162 & 278.578 & 284.304 & 400.997 & 745.917 & 392.37 & 459.794 & 449.066 & 439.107 \\
\hline $\mathrm{Y}$ & 32.571 & 34.541 & 26.345 & 31.324 & 25.871 & 37.396 & 34.726 & 38.713 & 35.625 \\
\hline $\mathrm{Zr}$ & 22.512 & 24.429 & 11.521 & 26.453 & 16.089 & 31.162 & 29.591 & 29.941 & 24.473 \\
\hline $\mathrm{Nb}$ & 11.802 & 12.814 & 10.554 & 20.228 & 21.04 & 22.203 & 26.393 & 24.433 & 17.372 \\
\hline Cs & 0.107 & 0.132 & 0.267 & 0.313 & 0.108 & 0.794 & 0.306 & 1.009 & 0.132 \\
\hline $\mathrm{Ba}$ & 64.343 & 73.808 & 52.09 & 157.013 & 1231.287 & 138.371 & 233.288 & 190.707 & 220.273 \\
\hline $\mathrm{La}$ & 14.796 & 15.728 & 14.083 & 22.541 & 24.891 & 25.55 & 32.158 & 27.022 & 32.152 \\
\hline $\mathrm{Ce}$ & 35.06 & 37.294 & 32.584 & 50.229 & 52.079 & 56.596 & 66.579 & 60.171 & 69.449 \\
\hline $\operatorname{Pr}$ & 4.283 & 4.577 & 3.876 & 5.864 & 5.677 & 6.562 & 7.371 & 7.009 & 7.763 \\
\hline $\mathrm{Nd}$ & 24.494 & 25.897 & 21.695 & 31.978 & 29.42 & 35.766 & 38.214 & 38.466 & 41.485 \\
\hline $\mathrm{Sm}$ & 6.029 & 6.574 & 5.361 & 7.505 & 6.302 & 8.314 & 8.099 & 8.792 & 8.877 \\
\hline $\mathrm{Eu}$ & 1.981 & 2.114 & 1.747 & 2.347 & 2.436 & 2.626 & 2.581 & 2.856 & 2.796 \\
\hline $\mathrm{Gd}$ & 7.229 & 7.829 & 6.371 & 8.734 & 7.363 & 9.913 & 9.873 & 10.496 & 10.303 \\
\hline $\mathrm{Tb}$ & 1.161 & 1.241 & 0.994 & 1.295 & 1.031 & 1.462 & 1.367 & 1.546 & 1.466 \\
\hline Dy & 5.54 & 5.992 & 4.598 & 5.768 & 4.627 & 6.815 & 6.139 & 7.125 & 6.376 \\
\hline Ho & 1.104 & 1.197 & 0.921 & 1.098 & 0.876 & 1.303 & 1.186 & 1.388 & 1.229 \\
\hline Er & 3.339 & 3.594 & 2.695 & 3.394 & 2.599 & 3.952 & 3.54 & 4.174 & 3.762 \\
\hline $\mathrm{Tm}$ & 0.509 & 0.548 & 0.403 & 0.501 & 0.388 & 0.601 & 0.541 & 0.612 & 0.544 \\
\hline $\mathrm{Yb}$ & 2.679 & 2.836 & 2.027 & 2.473 & 1.914 & 3.013 & 2.687 & 3.113 & 2.741 \\
\hline $\mathrm{Lu}$ & 0.384 & 0.4 & 0.301 & 0.358 & 0.279 & 0.424 & 0.392 & 0.443 & 0.413 \\
\hline $\mathrm{Hf}$ & 0.681 & 0.747 & 0.417 & 0.812 & 0.551 & 0.967 & 0.899 & 0.974 & 0.773 \\
\hline $\mathrm{Ta}$ & 0.836 & 0.951 & 0.725 & 1.442 & 1.392 & 1.621 & 1.821 & 1.843 & 1.341 \\
\hline $\mathrm{Pb}$ & 1.842 & 2.12 & 2.39 & 2.591 & 2.724 & 2.489 & 2.733 & 3.015 & 3.196 \\
\hline Th & 1.703 & 1.843 & 1.337 & 2.748 & 3.12 & 3.102 & 3.865 & 3.249 & 5.23 \\
\hline $\mathrm{U}$ & 0.269 & 0.284 & 0.171 & 0.3 & 0.282 & 0.411 & 0.416 & 0.434 & 0.874 \\
\hline $\mathrm{La} / \mathrm{Yb}$ & 5.522956 & 5.545839 & 6.947706 & 9.11484 & 13.0047 & 8.47992 & 11.96799 & 8.680373 & 11.73003 \\
\hline $\mathrm{Th} / \mathrm{Ta}$ & 2.037081 & 1.93796 & 1.844138 & 1.905687 & 2.241379 & 1.913634 & 2.12246 & 1.762887 & 3.900075 \\
\hline $\mathrm{Th} / \mathrm{Yb}$ & 0.635685 & 0.649859 & 0.659595 & 1.111201 & 1.630094 & 1.029539 & 1.438407 & 1.043688 & 1.908063 \\
\hline $\mathrm{Nb} / \mathrm{Yb}$ & 4.405375 & 4.518336 & 5.206709 & 8.179539 & 10.99269 & 7.369067 & 9.822479 & 7.848699 & 6.337833 \\
\hline $\mathrm{Nb} / \mathrm{Th}$ & 6.930123 & 6.952794 & 7.893792 & 7.36099 & 6.74359 & 7.15764 & 6.828719 & 7.52016 & 3.321606 \\
\hline
\end{tabular}


Table 2. (Continued.)

\begin{tabular}{|c|c|c|c|c|c|c|c|c|c|c|}
\hline $\begin{array}{l}\text { Flow no. } \\
\text { Sample }\end{array}$ & $\begin{array}{c}10 \\
\mathrm{~F}-20\end{array}$ & $\begin{array}{c}11 \\
\mathrm{~F}-21\end{array}$ & $\begin{array}{c}12 \\
\mathrm{~F}-24\end{array}$ & $\begin{array}{c}13 \\
\mathrm{~F}-26\end{array}$ & $\begin{array}{c}14 \\
\text { F-34B }\end{array}$ & $\begin{array}{c}15 \\
\text { F-36 }\end{array}$ & $\begin{array}{c}16 \\
\mathrm{~F}-44\end{array}$ & $\begin{array}{c}17 \\
\mathrm{~F}-48\end{array}$ & $\begin{array}{c}18 \\
\text { F-54 }\end{array}$ & $\begin{array}{c}19 \\
\text { F-55 }\end{array}$ \\
\hline $\mathrm{SiO}_{2}$ & 48.7 & 48.6 & 52.7 & 46.7 & 53.4 & 49.6 & 46.8 & 51.5 & 53.7 & 46.8 \\
\hline $\mathrm{Al}_{2} \mathrm{O}_{3}$ & 12.55 & 13.2 & 12.51 & 12.76 & 11.94 & 11.92 & 13.81 & 12.33 & 12.79 & 12.53 \\
\hline $\mathrm{Fe}_{2} \mathrm{O}_{3}$ & 14.43 & 11.46 & 13.55 & 14.19 & 13 & 12.33 & 13.34 & 14.34 & 12.26 & 14.59 \\
\hline $\mathrm{MnO}$ & 0.13 & 0.11 & 0.12 & 0.13 & 0.12 & 0.12 & 0.1 & 0.12 & 0.13 & 0.15 \\
\hline $\mathrm{MgO}$ & 6.39 & 9.5 & 4.48 & 8.97 & 6.15 & 7.64 & 8.8 & 6.68 & 5.49 & 8.73 \\
\hline $\mathrm{CaO}$ & 9.61 & 10.36 & 8.54 & 10.3 & 7.47 & 9.22 & 9.77 & 6.52 & 6.88 & 10.1 \\
\hline $\mathrm{Na}_{2} \mathrm{O}$ & 2.38 & 2.11 & 2.6 & 2.35 & 2.53 & 2.62 & 2.44 & 2.17 & 2.95 & 2.31 \\
\hline $\mathrm{K}_{2} \mathrm{O}$ & 0.7 & 0.83 & 0.93 & 0.69 & 1.16 & 1.12 & 0.74 & 1.22 & 1.26 & 0.68 \\
\hline $\mathrm{TiO}_{2}$ & 3.17 & 2.05 & 2.79 & 2.47 & 2.75 & 3.03 & 2.49 & 2.97 & 2.72 & 2.3 \\
\hline $\mathrm{P}_{2} \mathrm{O}_{5}$ & 0.53 & 0.2 & 0.41 & 0.23 & 0.32 & 1 & 0.29 & 0.259 & 0.32 & 0.21 \\
\hline $\mathrm{Sc}$ & 28.779 & 32.368 & 28.937 & 36.679 & 28.896 & 25.915 & 27.701 & 26.651 & 25.638 & 33.197 \\
\hline V & 324.087 & 313.382 & 433.218 & 390.081 & 380.890 & 302.644 & 317.332 & 362.959 & 274.16 & 369.622 \\
\hline $\mathrm{Cr}$ & 42.323 & 48.777 & 42.724 & 90.159 & 52.589 & 49.936 & 58.918 & 33.082 & 37.771 & 62.615 \\
\hline $\mathrm{Co}$ & 43.054 & 51.974 & 41.406 & 49.422 & 45.328 & 39.319 & 52.874 & 41.223 & 29.489 & 47.278 \\
\hline $\mathrm{Ni}$ & 11.185 & 13.708 & 9.333 & 29.521 & 13.823 & 11.403 & 21.855 & 6.482 & 4.832 & 18.438 \\
\hline $\mathrm{Cu}$ & 59.241 & 49.676 & 99.534 & 167.763 & 115.839 & 169.191 & 57.781 & 29.623 & 22.396 & 93.628 \\
\hline $\mathrm{Zn}$ & 101.131 & 71.664 & 117.486 & 82.553 & 95.23 & 134.12 & 87.875 & 83.244 & 102.109 & 70.568 \\
\hline $\mathrm{Ga}$ & 22.464 & 20.746 & 22.336 & 20.106 & 22.241 & 25.599 & 21.175 & 20.048 & 21.476 & 21.867 \\
\hline $\mathrm{Rb}$ & 26.043 & 20.36 & 23.796 & 12.273 & 61.109 & 42.43 & 10.475 & 58.908 & 74.117 & 12.108 \\
\hline $\mathrm{Sr}$ & 545.544 & 381.076 & 446.072 & 290.75 & 346.208 & 666.176 & 450.609 & 326.795 & 363.636 & 324.855 \\
\hline Y & 36.886 & 24.726 & 43.125 & 29.608 & 39.359 & 56.299 & 26.479 & 30.494 & 35.767 & 30.233 \\
\hline $\mathrm{Zr}$ & 24.894 & 20.051 & 37.76 & 16.466 & 34.04 & 63.306 & 21.677 & 26.94 & 33.092 & 6.715 \\
\hline $\mathrm{Nb}$ & 18.117 & 13.775 & 27.754 & 11.73 & 24.847 & 84.178 & 15.161 & 16.575 & 21.43 & 10.409 \\
\hline Cs & 2.333 & 0.237 & 2.003 & 0.121 & 0.333 & 0.503 & 1.268 & 1.124 & 0.915 & 0.193 \\
\hline $\mathrm{Ba}$ & 190.981 & 151.746 & 242.672 & 77.891 & 241.876 & 351.657 & 133.042 & 310.445 & 233.062 & 119.864 \\
\hline $\mathrm{La}$ & 33.663 & 21.401 & 49.198 & 15.19 & 37.666 & 97.871 & 22.299 & 28.989 & 34.907 & 14.977 \\
\hline $\mathrm{Ce}$ & 72.541 & 45.375 & 97.41 & 35.608 & 78.931 & 202.974 & 48.119 & 60.177 & 73.206 & 34.721 \\
\hline $\operatorname{Pr}$ & 8.194 & 5.104 & 10.746 & 4.328 & 8.7 & 21.627 & 5.327 & 6.758 & 8.013 & 4.195 \\
\hline $\mathrm{Nd}$ & 44.136 & 26.881 & 54.079 & 24.912 & 45.468 & 107.289 & 28.463 & 34.885 & 40.833 & 24.335 \\
\hline $\mathrm{Sm}$ & 9.353 & 5.832 & 10.746 & 6.215 & 9.659 & 19.699 & 6.363 & 7.551 & 8.544 & 6.116 \\
\hline $\mathrm{Eu}$ & 2.936 & 1.906 & 3.067 & 2.027 & 2.834 & 5.321 & 1.986 & 2.217 & 2.55 & 2.006 \\
\hline Gd & 10.951 & 6.875 & 12.764 & 7.487 & 11.589 & 22.158 & 7.39 & 8.673 & 9.734 & 7.371 \\
\hline $\mathrm{Tb}$ & 1.546 & 1.014 & 1.794 & 1.182 & 1.609 & 2.801 & 1.052 & 1.243 & 1.399 & 1.178 \\
\hline Dy & 6.714 & 4.494 & 7.517 & 5.593 & 7.128 & 11.078 & 4.809 & 5.565 & 6.238 & 5.67 \\
\hline Ho & 1.295 & 0.851 & 1.519 & 1.076 & 1.373 & 2.025 & 0.95 & 1.077 & 1.233 & 1.087 \\
\hline Er & 3.941 & 2.607 & 4.656 & 3.172 & 4.134 & 6.189 & 2.874 & 3.309 & 3.757 & 3.141 \\
\hline $\mathrm{Tm}$ & 0.579 & 0.391 & 0.685 & 0.481 & 0.62 & 0.848 & 0.433 & 0.514 & 0.584 & 0.457 \\
\hline $\mathrm{Yb}$ & 2.897 & 2.016 & 3.632 & 2.393 & 3.235 & 4.12 & 2.222 & 2.626 & 2.929 & 2.182 \\
\hline $\mathrm{Lu}$ & 0.418 & 0.29 & 0.526 & 0.328 & 0.469 & 0.599 & 0.326 & 0.369 & 0.448 & 0.295 \\
\hline $\mathrm{Hf}$ & 0.785 & 0.649 & 1.182 & 0.549 & 1.159 & 1.868 & 0.692 & 0.883 & 1.043 & 0.251 \\
\hline $\mathrm{Ta}$ & 1.445 & 1.087 & 1.858 & 0.882 & 1.876 & 7.135 & 1.128 & 1.269 & 1.678 & 0.822 \\
\hline $\mathrm{Pb}$ & 2.955 & 2.826 & 3.093 & 2.334 & 3.512 & 2.692 & 2.284 & 4.054 & 3.231 & 2.527 \\
\hline Th & 5.452 & 3.579 & 8.03 & 1.276 & 5.413 & 11.779 & 3.42 & 6.425 & 6.789 & 1.338 \\
\hline $\mathrm{U}$ & 0.824 & 0.556 & 1.139 & 0.21 & 0.823 & 1.448 & 0.456 & 0.937 & 1.012 & 0.191 \\
\hline $\mathrm{La} / \mathrm{Yb}$ & 11.619 & 10.6155 & 13.5457 & 6.34768 & 11.6432 & 23.7551 & 10.0355 & 11.0392 & 11.9177 & 6.86388 \\
\hline $\mathrm{Th} / \mathrm{Ta}$ & 3.7730 & 3.29254 & 4.32185 & 1.44671 & 2.88539 & 1.65087 & 3.03191 & 5.06304 & 4.04588 & 1.62773 \\
\hline $\mathrm{Th} / \mathrm{Yb}$ & 1.8819 & 1.77529 & 2.21090 & 0.53322 & 1.67326 & 2.85898 & 1.53915 & 2.44668 & 2.31785 & 0.61319 \\
\hline $\mathrm{Nb} / \mathrm{Yb}$ & 6.2537 & 6.83283 & 7.64152 & 4.90179 & 7.68068 & 20.4315 & 6.82313 & 6.31188 & 7.31649 & 4.77039 \\
\hline $\mathrm{Nb} / \mathrm{Th}$ & 3.3230 & 3.84884 & 3.45628 & 9.19279 & 4.59024 & 7.14644 & 4.43304 & 2.57976 & 3.15657 & 7.77952 \\
\hline
\end{tabular}




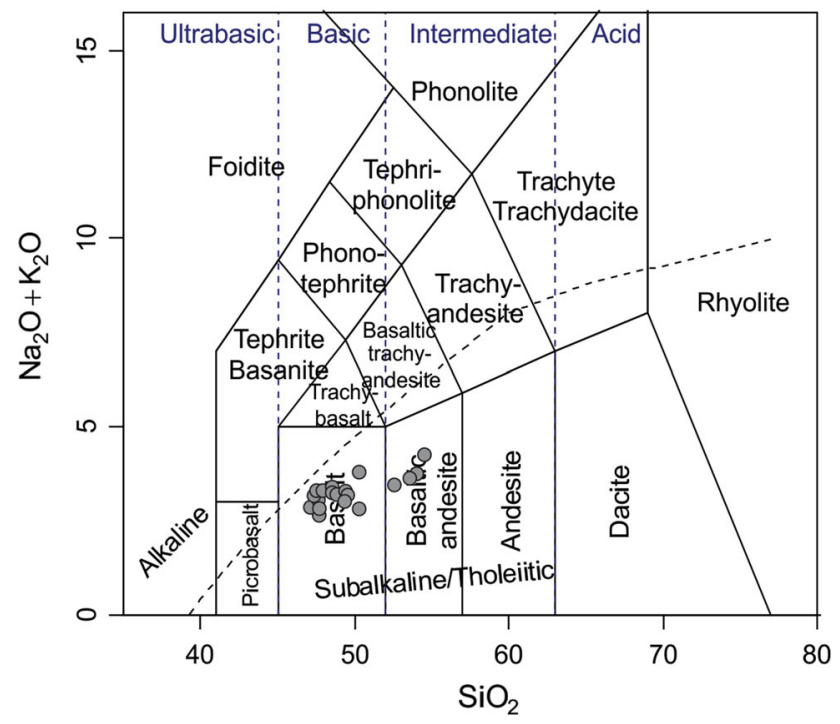

Figure 4. Total alkali $\left(\mathrm{Na}_{2} \mathrm{O}+\mathrm{K}_{2} \mathrm{O}\right)$ vs. silica $\left(\mathrm{SiO}_{2}\right)$ diagram (after Le Bas et al. 1986) showing the samples from the Chhotaudepur region in the field of basalt and basaltic andesite.

of the carbonated silicate parental magma during concurrent fractional crystallization of silicates and exsolution of carbonate melt. Sheth and Ray (2002) developed an AFC model in terms of Sr concentration and Sr isotopic compositions to explain the genesis of the magmatic rocks of Pavagadh area using the western Indian granite as a contaminant. Later on, Sheth and Melluso (2008) discussed the importance of AFC in the genesis of the magmatic rocks of Pavagadh volcanic suite. Hari et al. (2011) proposed that gabbro and orthopyroxene gabbro from Phenaimata igneous complex, part of the Chhotaudepur province were a product of AFC.

In order to evaluate the significance of $\mathrm{AFC}$ process in the modification of the magma of Chhotaudepur province, $(\mathrm{Nb} / \mathrm{Th})_{\mathrm{PM}}$ vs. $(\mathrm{Th} / \mathrm{Yb})_{\mathrm{PM}}$ diagram was employed (figure 7a). It is generally accepted that mantle melt compositions have high $\mathrm{Nb} / \mathrm{Th}$ values and low $\mathrm{Th} / \mathrm{Yb}$ values, whereas on the other hand, low $(\mathrm{Nb} / \mathrm{Th})_{\mathrm{PM}}$ values and high $(\mathrm{Th} / \mathrm{Yb})_{\mathrm{PM}}$ values are considered to be derived as the result of assimilation of crustal material (Lightfoot and Hawkesworth 1988; Lightfoot et al. 1990; Dessai et al. 2008). The basalts and basaltic andesites of the Chhotaudepur area plot close to a consistent mixing trend between typical mantle composition (i.e., close to 1) and crustal contaminants, indicating that the magmas of the Chhotaudepur region were assimilated with a significant amount of crustal components prior to

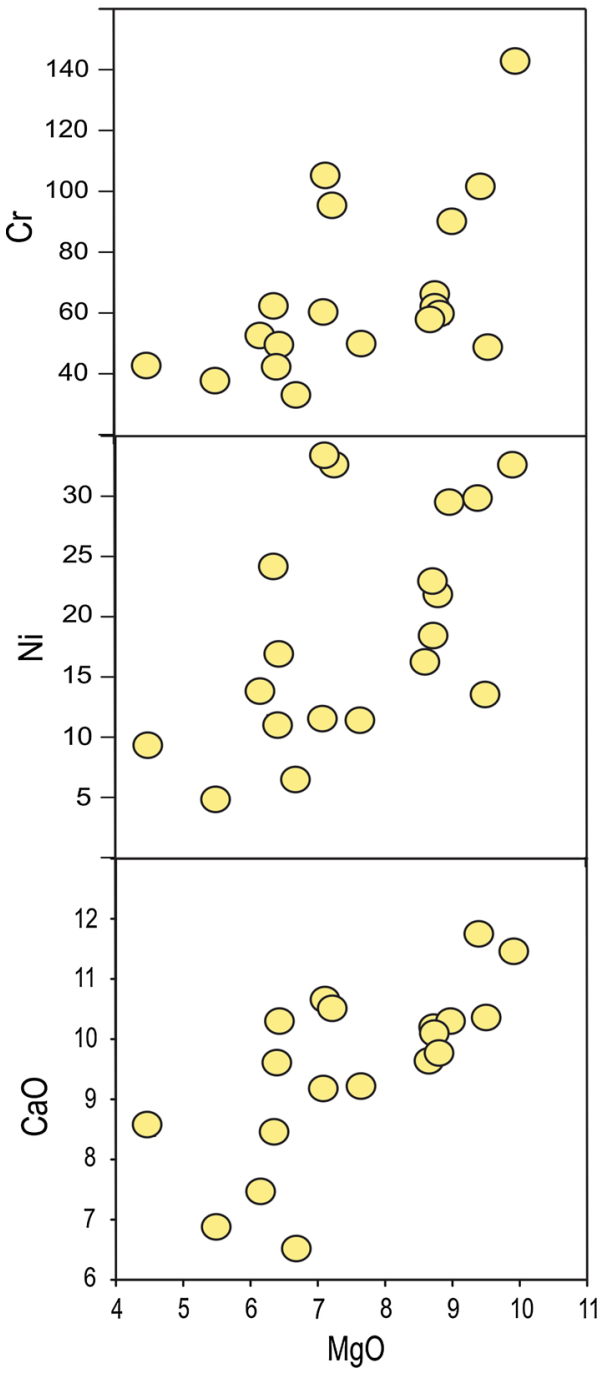

Figure 5. Variation diagrams of $\mathrm{Cr}, \mathrm{Ni}$, and $\mathrm{CaO}$ with $\mathrm{MgO}$ of basalt and basaltic andesite flows from the Chhotaudepur region.

their emplacement and eruption. The Kalasubai Subgroup data also exhibit a similar trend of Chhotaudepur samples. On the other hand, Wai and Lonavala subgroup magmas have lower assimilation contents (figure $7 \mathrm{a}$ ). In the $\mathrm{Th} / \mathrm{Yb}$ vs. $\mathrm{Nb} / \mathrm{Yb}$ diagram (figure $7 \mathrm{~b}$ ) the field of Reunion basalts represents plume asthenosphere with the variable incorporation of the depleted upper mantle. The basaltic rocks of Chhotaudepur region plot close to the field of Reunion plume and the mantle array. The displacement of the suite is noticeable towards the direction of crustal contamination. Deccan tholeiites and alkaline felsic rocks also exhibit a significant trend of crustal contamination. The marked variations in $\mathrm{Rb}(2.4-74.11$ $\mathrm{ppm}$ ) values and varying $\mathrm{La} / \mathrm{Yb}(5.5-23.7)$ ratios in the studied samples also suggest the possibility of crustal contamination. The OIB, continental 

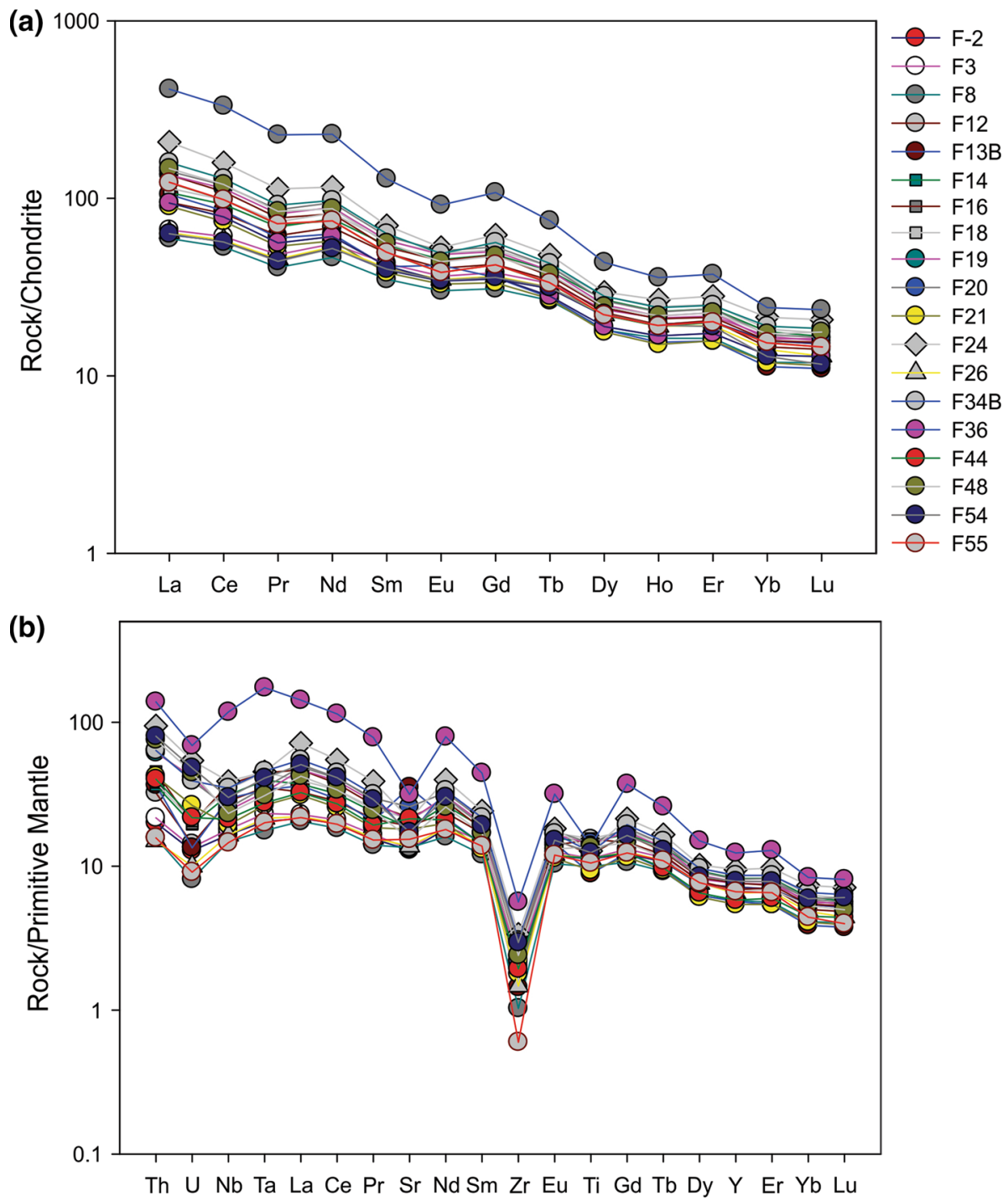

Figure 6. (a) Chondrite normalized REE distribution patterns and (b) primitive mantle normalized multi-element spider diagram for basalt and basaltic andesite flows from Chhotaudepur province, of DLIP (normalization values are from Sun and McDonough 1989).

alkali basalts and kimberlites have $\mathrm{La} / \mathrm{Yb}<1$, whereas the continental flood basalts show wider variation in $\mathrm{La} / \mathrm{Yb}$ ratio $(0.5-7)$ (McDonough and Sun 1995). In the present case, La/Yb values range from 5.5 to 23.7 indicating that basaltic magmas in the Chhotaudepur region have been contaminated by crustal materials during their ascent and emplacement. It is generally considered that positive Th peaks in the primitive mantle normalized multi-element diagram along with variable $\mathrm{Th} / \mathrm{Ta}$ ratios (1.4-5.06) also point towards crustal contamination of the samples. Further, low and variable $\mathrm{Nb} / \mathrm{Th}$ ratio basaltic flows of Chhotaudepur region (2.5-7.89) also indicate crustal contamination as $\mathrm{Nb} / \mathrm{Th}$ ratio of the primitive mantle is 8 and the continental crust is $\sim 1.1$ (Taylor and McLennan 1985).
When the samples of Chhotaudepur region were plotted in the $\mathrm{Th} / \mathrm{Ta}$ vs. $\mathrm{La} / \mathrm{Yb}$ diagram (figure $8 \mathrm{a}$ ), most of the data plot above the mantle component portion. The rise in $\mathrm{Th} / \mathrm{Ta}$ and $\mathrm{La} / \mathrm{Yb}$ ratios and the linear trend of the samples indicate the involvement of AFC processes in the genesis of these rocks (Condie 1997). Defant and Nielsen (1990) suggested that AFC processes can elevate $\mathrm{Th} / \mathrm{Ta}$ and $\mathrm{La} / \mathrm{Yb}$ ratios by up to a factor of 3 or 4 in the case of upper continental crust and a factor of 2 for the lower continental crust. From figure 8(a), it is evident that the Deccan lavas of Kalasubai, Lonavala, and Chhotaudepur region were dominantly contaminated by the continental crust and the samples of Wai Subgroup, which plot close to the FOZO zone are with less contamination. 

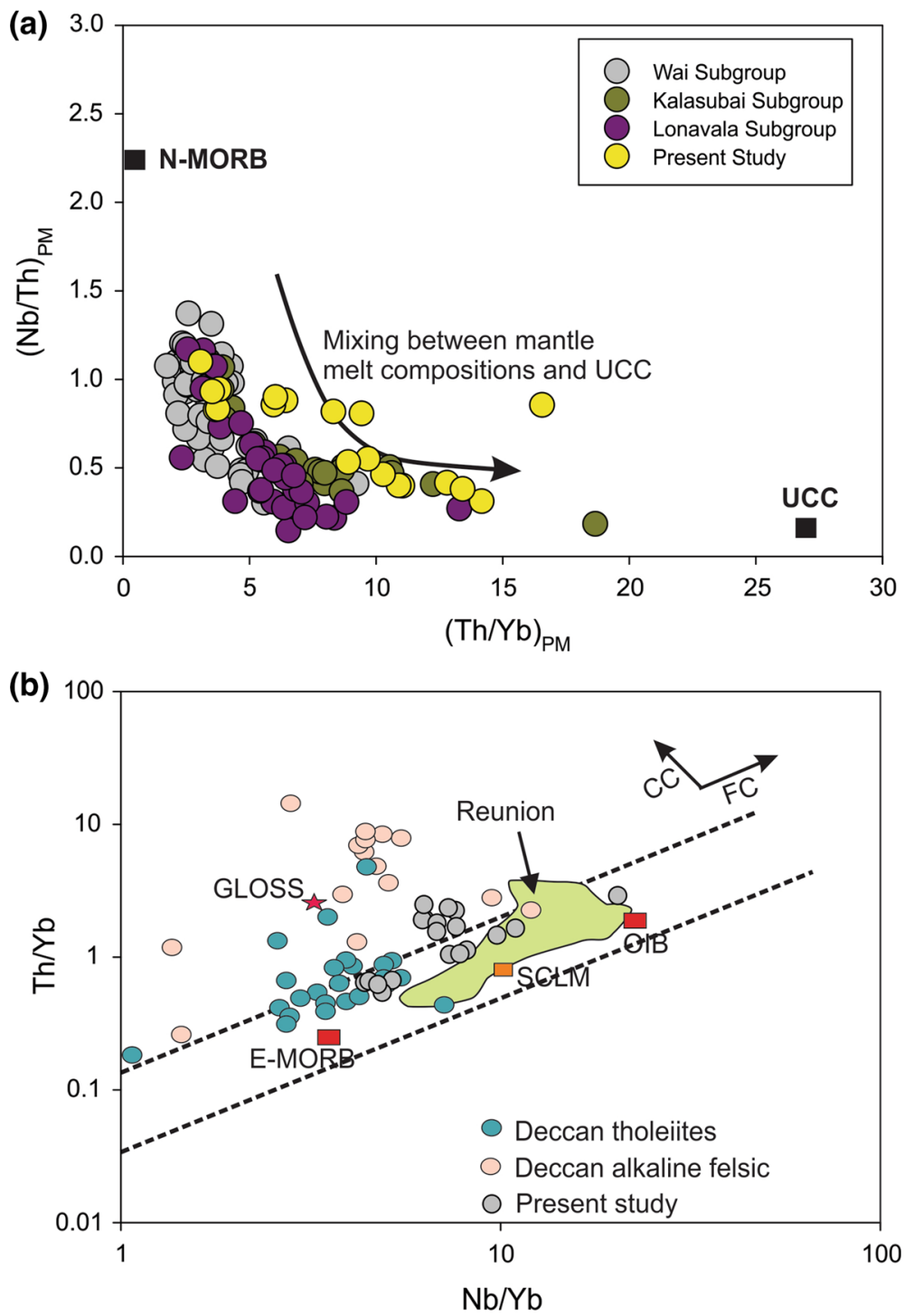

Figure 7. (a) $(\mathrm{Nb} / \mathrm{Th})_{\mathrm{PM}}$ vs. $(\mathrm{Th} / \mathrm{Yb})_{\mathrm{PM}}$ plot showing variations in the $(\mathrm{Nb} / \mathrm{Th})_{\mathrm{PM}}$ and $(\mathrm{Th} / \mathrm{Yb})_{\mathrm{PM}} \mathrm{ratios}$ in samples from the Chhotaudepur region, Wai Subgroup, Kalasubai Subgroup and Lonavala Subgroup. Data for the Wai, Kalasubai and Lonavala Subgroups are from the GEOROCK database (http://georoc.mpch-mainz.gwdg.de/georoc/). The ratios are normalized to primitive mantle values of Sun and McDonough (1989), N-MORB composition is from Hoffman (1988) and average Upper Continental Crust (UCC) composition is from Taylor and McLennan (1985). (b) Th/Yb vs. Nb/Yb diagram of the flows of Chhotaudepur region compared to the Deccan tholeiitic basalts (Lightfoot and Hawkesworth 1988; Chatterjee and Bhattacharji 2001; Sheth et al. 2004), Deccan alkaline felsic rocks (Chatterjee and Bhattacharji 2001), and modern-day Réunion plume basalts (Fisk et al. 1988; Paul et al. 2005). N-MORB, E-MORB, and OIB (Ocean Island Basalt) compositions are from Sun and McDonough (1989). SCLM composition is from McDonough (1990); GLOSS from Plank and Langmuir (1998). Vectors CC and FC approximate the direction of crustal contamination and fractional crystallization, respectively. Dashed lines indicate the mantle array (Pearce 2008).

The $(\mathrm{Th} / \mathrm{Yb})_{\mathrm{PM}}$ vs. $(\mathrm{Nb} / \mathrm{Yb})_{\mathrm{PM}}$ diagram (figure $8 \mathrm{~b}$ ) is generally used to elucidate the difference between crustal contamination and variation in the melt sourcing. The $(\mathrm{Th} / \mathrm{Yb})_{\mathrm{PM}}$ and $(\mathrm{Nb} / \mathrm{Yb})_{\mathrm{PM}}$ ratios of enriched mantle sources EMI, EMII, HIMU (Condie 1997) and upper continental crust (Taylor and McLennan 1985) were compared with samples of DLIP. From the $(\mathrm{Th} / \mathrm{Yb})_{\mathrm{PM}}$ vs. $(\mathrm{Nb} / \mathrm{Yb})_{\mathrm{PM}}$ diagram, it can be deciphered that the magma compositions of DLIP are controlled by a mixture of source enrichment and crustal contaminant. Therefore, it can be presumed that AFC was one of the most important processes which were involved in the modification of the magma flows in Chhotaudepur region. 

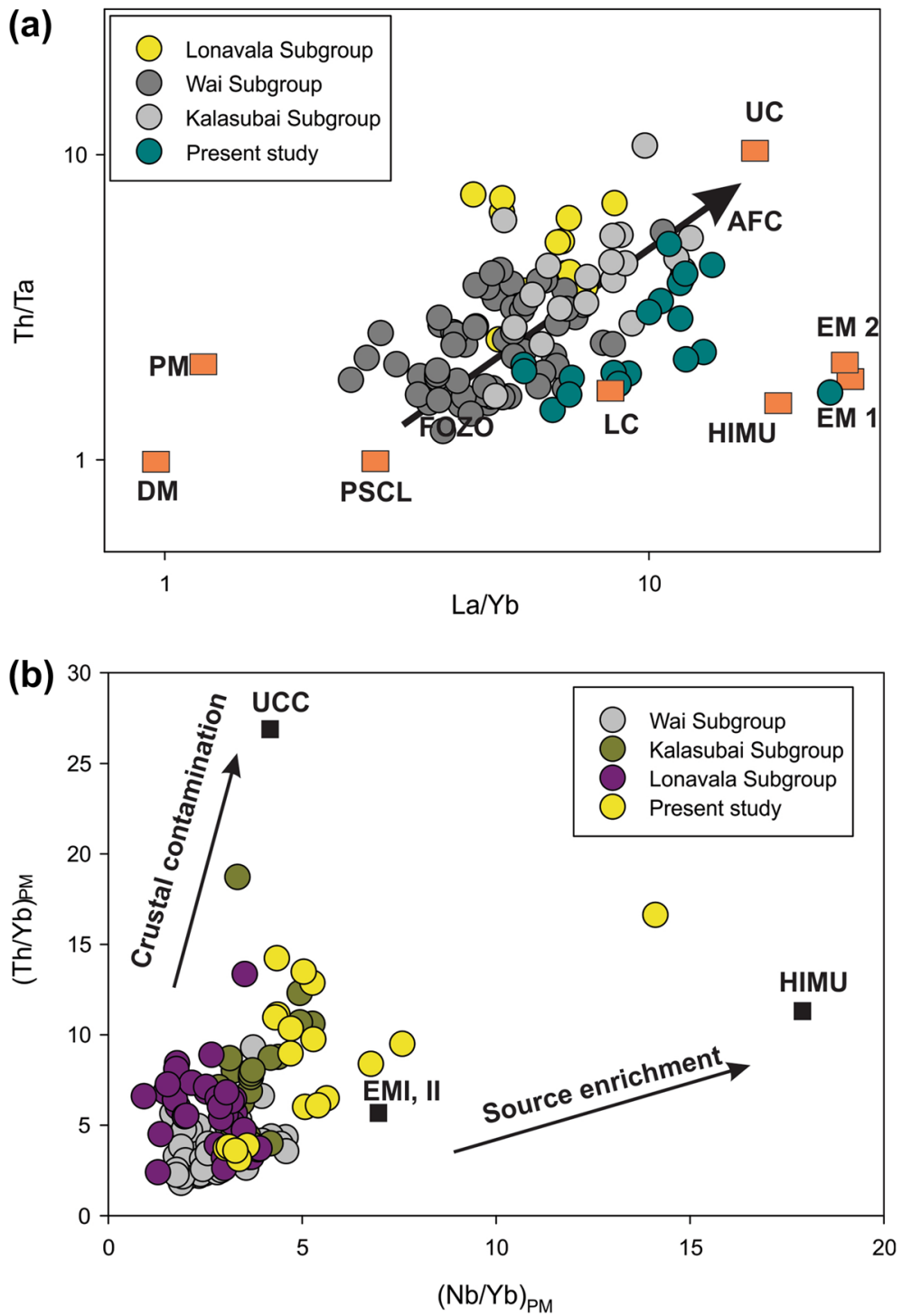

Figure 8. (a) Th/Ta vs. La/Yb plot (Condie 1997) showing plots of Chhotaudepur region, Wai Subgroup, Kalasubai Subgroup, Lonavala Subgroup and the distribution of mantle components. DM: depleted mantle, PM: primitive mantle, PSCL: post-Archean subcontinental lithosphere, LC: lower continental crust, UC: upper continental crust, HIMU: high U/Pb mantle source, EM 1 and EM 2: enriched mantle sources, FOZO: lower mantle plume component (region on the graph beneath and around the word FOZO), AFC: assimilation and fractional crystallization trend. Flows from the Chhotaudepur region of DLIP show an AFC trend towards upper crust. (b) (Th/Yb) PM $_{\text {vs. }}(\mathrm{Nb} / \mathrm{Yb})_{\mathrm{PM}}$ plot showing variations in the $(\mathrm{Th} / \mathrm{Yb})_{\mathrm{PM}}$ and $(\mathrm{Nb} / \mathrm{Yb})_{\mathrm{PM}}$ ratios of samples from the Chhotaudepur region, Wai Subgroup, Kalasubai Subgroup and Lonavala Subgroup. Ratios are normalized to primitive mantle values of Sun and McDonough (1989). N-MORB composition is from Hofmann (1988), upper continental crust (UCC) composition is from Taylor and McLennan (1985), and enriched mantle (EMI, EMII) and HIMU values are from Condie (1997). Data for the Wai, Kalasubai and Lonavala Subgroups are from the GEOROCK database (http://georoc.mpch-mainz.gwdg.de/georoc/).

\subsection{AFC modelling of the flows}

The role of crustal contamination and the depth of magmatic sources are two important parameters required to understand the emplacement mechanisms of a Large Igneous Province (LIP) (Wood 1980; Rudnick et al. 1986; Arndt et al. 1993; Peng et al. 1994; Chesley and Ruiz 1998;
Arndt et al. 2000; Xiao et al. 2004; Tegner et al. 2005). Major and trace element concentrations of this study suggest that the Deccan magmas have suffered a significant amount of fractional crystallization coupled with crustal assimilation. Assimilation and fractional crystallization (AFC), which is an important process for the modification of the magma is evaluated by considering $20 \%$ 
olivine, $25 \%$ clinopyroxene, $45 \%$ plagioclase and $10 \%$ titano-magnetite as the fractionating minerals. Mineral/melt partition coefficient values are taken after Claeson and Meurer (2004). The contaminant is taken as the average upper continental crust and the values are taken from Rudnick and Gao (2013). As the primitive magma (picrite) reported by Hari and Swarnkar (2011) from this region exhibit high incompatible elemental concentrations, thus, that rock could not be considered as the primary source of the flows of this region. In order to find out the primary rock, the method of Hofmann and Feigenson (1983) for calculating the parental magma from the differentiated one is adopted. The method involves the addition of minerals that supposedly fractionated from the parental magma. Once the composition of the parental melt was calculated, the calculations for AFC were carried out with the equation by DePaolo (1981) and Powell (1984)

$$
\left.C_{L}=C_{L}^{o} \cdot f+(r / r-1+D) \cdot C^{*} \cdot 1-f\right)
$$

where $C_{L}^{o}$ is the concentration of the trace element in original magma, $C^{*}$ is concentration of trace element in the contaminant, $r$ is the ratio of rate of assimilation to rate of fractional crystallization, $D$ is the bulk distribution coefficient for the fractionating assemblage, $f$ is $F^{[(r-1+D) /(r-1)]}$ and $F$ is fraction of magma remaining.

Keeping the rate of assimilation to fractional crystallization $(r)$ as 0.3 , the binary plotting was carried out and are presented in figures $9(\mathrm{a}-\mathrm{c})$. It has been found that the samples of the flows, plot within $r=0$ (perfect fractional crystallization) and $r=0.3$ indicating that these rocks are a product of AFC.

Condie (1997) proposed that the high and low$\mathrm{Ti}$ basalts of DLIP are derived from a mantle plume that interacted with the continental lithosphere. While tracing the source region characteristics of DLIP, Karmalkar et al. (2008) proposed that the magma underplating as a common phenomenon in the Kutch region and the magma chambers beneath the Kutch are connected by a network of interconnected dykes, from which the basaltic magmas periodically erupted. Vijaya Kumar et al. (2010) argued that the parental melts of DLIP have gone through different stages of fractional crystallization and were segregated within the lower crustal magma chamber(s) and underwent first stage fractional crystallization (a)

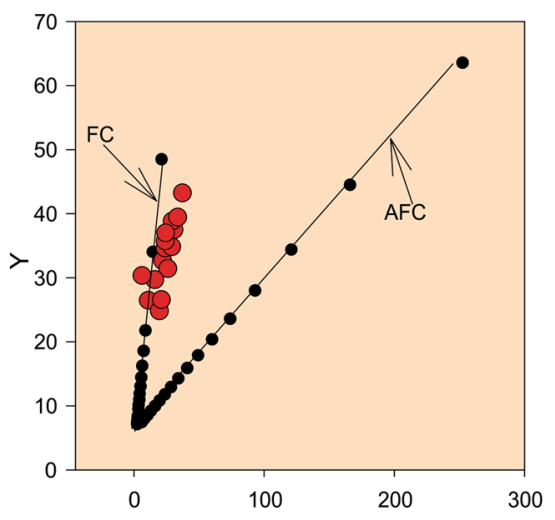

(b)

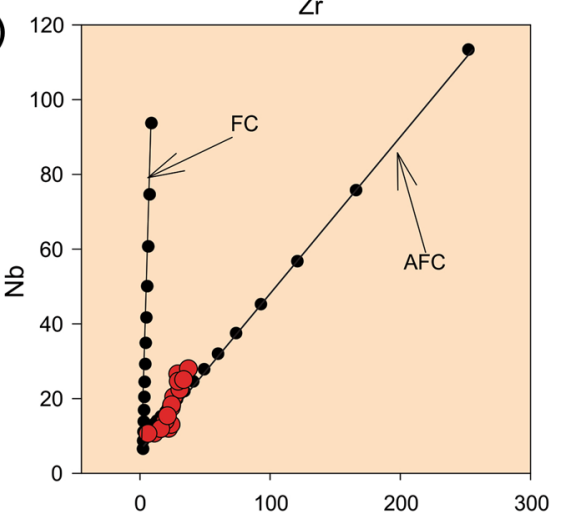

(c)

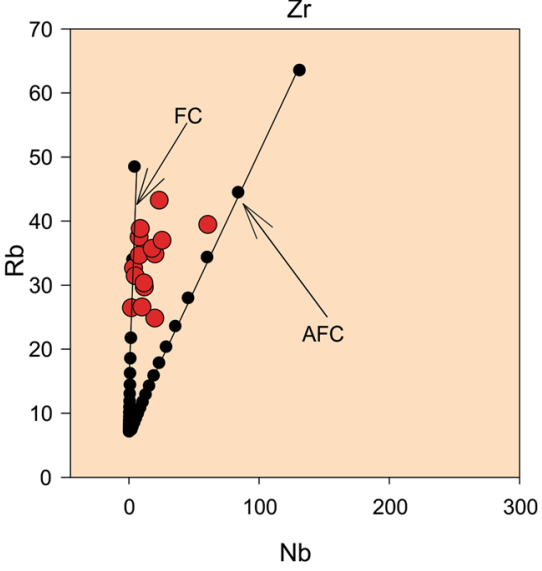

Figure 9. (a) Zr vs. $\mathrm{Y}$, (b) $\mathrm{Zr}$ vs. $\mathrm{Nb}$, and (c) $\mathrm{Nb}$ vs. $\mathrm{Rb}$ plots of the basalts and basaltic andesite flows of Chhotaudepur province. Liquid evolution curves were calculated for both fractional crystallization (FC) and concurrent assimilation and fractional crystallization (AFC). Each tick in the fractionation curve indicates $10 \%$ fractionation. The rate of assimilation to fractional crystallization $(r)$ is considered as 0.3 . Note that all the plotting of basalt and basaltic andesite fall within FC and AFC lines indicating that these rocks are a product of concurrent assimilation and fractional crystallization.

dominated by olivine and clinopyroxene ( \pm spinel), thereby producing Fe- and Al-rich tholeiitic melts. These fractionated melts migrated from the asthenospheric depths to the upper crustal regions and the melt again to get pounded in shallow-level 
magma chambers where concurrent assimilation and fractional crystallization took place.

\section{Conclusions}

None of the flow samples from Chhotaudepur province exhibit primary magma signatures as they have low $\mathrm{Mg} \#, \mathrm{Ni}$ (4.8-33.4 ppm) and $\mathrm{Cr}$ (33.08$143.06 \mathrm{ppm})$. The coherent chondrite normalized REE diagram and primitive mantle normalized multi-element diagram suggests a consanguinity among the flows. Low (Nb/Th) $\mathrm{PM}$ (0.30-1.09), high $(\mathrm{Th} / \mathrm{Yb})_{\mathrm{PM}}(3.09-16.58)$ ratios and marked variations in $\mathrm{Rb}(2.4-74.11 \mathrm{ppm})$ and variable $\mathrm{La} / \mathrm{Yb}$ (5.5-23.7) ratios suggests that magmas in the Chhotaudepur region were assimilated significantly by the crustal components to their emplacement and eruption. The elevated $\mathrm{Th} / \mathrm{Ta}$ and $\mathrm{La} / \mathrm{Yb}$ relation further indicate concurrent assimilation and fractional crystallization process were involved in the genesis of the flows. AFC modelling of the flows was carried out with $20 \%$ olivine, $25 \%$ clinopyroxene, $45 \%$ plagioclase and $10 \%$ titano-magnetite as fractionating minerals and upper continental crust as the assimilant. The result reveals that all the flows were modified by assimilation and fractional crystallization process.

\section{Acknowledgements}

We are thankful to Prof. N V Chalapathi Rao for his suggestions in improving the original manuscript. Helpful comments and reviews from Dr. Kirtikumar Randive and an anonymous reviewer are appreciated. Financial support from Department of Science and Technology (DST) to K R Hari is gratefully acknowledged.

\section{References}

Arndt N T, Czamanske G K, Wooden J L and Fedorenko V A 1993 Mantle and crustal contributions to continental flood volcanism; Tectonophys. 223 39-52, https://doi.org/10. 1016/0040-1951(93)90156-E.

Azzone R G, Munoz P M, Enrich G E, Alves A, Ruberti E and Gomes C B 2016 Petrographic, geochemical and isotopic evidence of crustal assimilation processes in the Ponte Nova alkaline mafic-ultramafic massif, SE Brazil; Lithos 260 58-75.

Basu A R, Renne P R, DasGupta D K, Teichmann F and Poreda R J 1993 Early and late alkali igneous pulses and a high- ${ }^{3} \mathrm{He}$ plume origin for the Deccan flood basalts; Science 261 902-905.
Beard J S, Ragland P C and Crawford M L 2005 Reactive bulk assimilation: A model for crust-mantle mixing in silicic magmas; Geology 33(8)681-684.

Bose M K 1972 Deccan basalts; Lithos 5(2) 131-145.

Chatterjee N and Bhattacharji S 2001 Origin of the felsic and basaltic dikes and flows in the Rajula-Palitana-Sihor area of the Deccan traps, Saurashtra, India: A geochemical and geochronological study; Int. Geol. Rev. 43(12) 10941116, https://doi.org/10.1080/00206810109465063.

Chenet A L, Quidelleur X, Fluteau F, Courtillot V and Bajpai $\mathrm{S} 2007{ }^{40} \mathrm{~K}-{ }^{40} \mathrm{Ar}$ dating of the main Deccan large igneous province: Further evidence of KTB age and short duration; Earth Planet. Sci. Lett. 263 1-15, https://doi. org/10.1016/j.epsl.2007.07.011.

Chesley J T and Ruiz J 1998 Crust-mantle interaction in large igneous provinces: Implications from the Re-Os isotope systematics of the Columbia River flood basalts; Earth Planet Sci. Lett. 154 1-11, https://doi.org/10. 1016/S0012-821X(97)00176-3.

Claeson D T and Meurer W P 2004 Fractional crystallization of hydrous basaltic 'arc-type' magmas and the formation of amphibole-bearing gabbroic cumulates; Contrib. Mineral. Petrol. 147(3) 288-304, https://doi.org/10. 1007/s00410-003-0536-0.

Coffin M F and Eldholm O 1994 Large igneous provinces: Crustal structure, dimensions, and external consequences; Rev. Geophys. 32(1) 1-36.

Collier J S, Sansom V, Ishizuka O, Taylor R N, Minshull T A and Whitmarsh R B 2008 Age of Seychelles-India break-up; Earth Planet. Sci. Lett. 272 264-277, https:// doi.org/10.1016/j.epsl.2008.04.045.

Condie K C 1997 Sources of Proterozoic mafic dyke swarms: Constraints from $\mathrm{Th} / \mathrm{Ta}$ and $\mathrm{La} / \mathrm{Yb}$ ratios; Precamb. Res. 81(1-2) 3-14, https://doi.org/10.1016/ S0301-9268(96)00020-4.

Courtillot V E and Renne P R 2003 On the ages of flood basalt events; Comptes. Rendus. Geosci. 335 113-140, https://doi.org/10.1016/S1631-0713(03)00006-3.

Cribb J W and Barton M 1996 Geochemical effects of decoupled fractional crystallization and crustal assimilation; Lithos 37(4) 293-307, https://doi.org/10.1016/ 0024-4937(95)00027-5.

Defant M J and Nielsen R L 1990 Interpretation of open system petrogenetic processes: Phase equilibria constraints on magma evolution; Geochim. Cosmochim. Acta 54 87102, https://doi.org/10.1016/0016-7037(90)90197-S.

DePaolo D J 1981 Trace element and isotopic effects of combined wallrock assimilation and fractional crystallization; Earth Planet. Sci. Lett. 53 189-202, https://doi.org/10. 1016/0012-821X(81)90153-9.

Dessai A G, Downes H, López-Moro F J and López-Plaza M 2008 Lower crustal contamination of Deccan Traps magmas: Evidence from tholeiitic dykes and granulite xenoliths from western India; Mineral. Petrol. 93(3-4) 243-272, https://doi.org/10.1007/s00710-007-0223-3.

Devey C W and Cox K G 1987 Relationships between crustal contamination and crystallisation in continental flood basalt magmas with special reference to the Deccan Traps of the Western Ghats, India; Earth Planet. Sci. Lett. 84 59-68, https://doi.org/10.1016/0012-821X(87)90176-2.

Ernst R and Bleeker W 2010 Large igneous provinces (LIPs), giant dyke swarms, and mantle plumes: Significance for 
breakup events within Canada and adjacent regions from 2.5 Ga to the Present; Can. J. Earth Sci. 47(5) 695-739, https://doi.org/10.1139/E10-025.

Fisk M R, Upton B G J, Ford C E and White W M 1988 Geochemical and experimental study of the genesis of magmas of Reunion Island, Indian Ocean; J. Geophys. Res. Solid Earth 93(B5) 4933-4950.

Freund S, Beier C, Krumm S and Haase K M 2013 Oxygen isotope evidence for the formation of andesitic-dacitic magmas from the fast-spreading Pacific-Antarctic Rise by assimilation-fractional crystallisation; Chem. Geol., https://doi.org/10.1016/j.chemgeo.2013.04.013.

Gallagher K and Hawkesworth C 1992 Dehydration melting and the generation of continental flood basalts; Nature 358(6381) 57-59.

Ganguly S, Ray J, Koeberl C, Ntaflos T and Banerjee M 2012 Mineral chemistry of lava flows from Linga area of the Eastern Deccan Volcanic Province, India; J. Earth Syst. Sci. 121(1) 91-108.

Ganguly S, Ray J, Koeberl C, Saha A, Thöni M and Balaram V 2014 Geochemistry and petrogenesis of lava flows around Linga, Chhindwara area in the Eastern Deccan Volcanic Province (EDVP), India; J. Asian Earth Sci. 91 174-193.

Genske F S, Beier C, Haase K M, Turner S P, Krumm S and Brandl P A 2013 Oxygen isotopes in the Azores islands: Crustal assimilation recorded in olivine; Geology 41(4) 491-494, https://doi.org/10.1130/G33911.1.

Greenough J D, Hari K R, Chatterjee A C and Santosh M 1998 Mildly alkaline basalts from Pavagadh Hill, India: Deccan flood basalts with an asthenospheric origin; Mineral. Petrol. 62(3) 223-245.

Grunder A L 1992 Two-stage contamination during crustal assimilation: Isotopic evidence from volcanic rocks in eastern Nevada; Contrib. Mineral. Petrol. 112(2) 219-229.

Hari K R 1998 Mineralogical and petrochemical studies of the lamprophyres around Chhaktalao area, Madhya Pradesh; J. Geol. Soc. India 51(1) 21-30.

Hari K R and Swarnkar V 2011 Petrogenesis of basaltic and doleritic dykes from Kawant, Chhotaudepur Province, Deccan Traps; Dyke Swarms: Keys for Geodynamic Interpretations, Springer Berlin Heidelberg, pp. 283-299.

Hari K R, Chalapathi Rao N V and Swarnkar V 2011 Petrogenesis of gabbro and orthopyroxene gabbro from the Phenai Mata Igneous Complex, Deccan volcanic province: Products of concurrent assimilation and fractional crystallization; J. Geol. Soc. India $\mathbf{7 8}$ 501-509.

Hari K R, Chalapathi Rao N V, Swarnkar V and Hou G 2014 Alkali feldspar syenites with shoshonitic affinities from Chhotaudepur area: Implication for mantle metasomatism in the Deccan large igneous province; Geosci. Front. 5 261-276.

Hari K R, Kumar M S, Santosh M and Rai S K 2000 Melt inclusions in olivine and pyroxene phenocrysts from lamprophyres of Chhaktalao area, Madhya Pradesh, India; J. Asian Earth Sci. 18(2) 155-161.

Hari K R, Nambiar C G, Furuyama K and Rai S K 2002 Some geochemical and petrogenetic relations between flows and dykes of Deccan Trap from Chhaktalao area, Madhya Pradesh; J. Geol. Soc. India 59(3) 225-232.
Hawkesworth C J, Rogers N W, Van Calsteren P W C and Menzies M A 1984 Mantle enrichment processes; Nature 311(5984) 331-335.

Hofmann A W 1988 Chemical differentiation of the Earth: The relationship between mantle, continental crust, and oceanic crust; Earth Planet. Sci. Lett. 90(3) 297-314.

Hofmann A W and Feigenson M D 1983 Case studies on the origin of basalt-I. Theory and reassessment of Grenada basalts; Contrib. Mineral. Petrol. 84(4) 390-405.

Iwamori H and Nakamura H 2015 Isotopic heterogeneity of oceanic, arc and continental basalts and its implications for mantle dynamics; Gondwana Res. 27(3) 1131-1152.

Jay A E, Mac Niocaill C, Widdowson M, Self S and Turner W 2009 New palaeomagnetic data from the Mahabaleshwar Plateau, Deccan Flood Basalt Province, India: Implications for the volcanostratigraphic architecture of continental flood basalt provinces; J. Geol. Soc. London 166(1) 13-24.

Jowitt S M and Ernst R E 2013 Geochemical assessment of the metallogenic potential of Proterozoic LIPs of Canada; Lithos 174 291-307.

Karmalkar N R, Kale M G, Duraiswami R A and Jonalgadda M 2008 Magma underplating and storage in the crustbuilding process beneath the Kutch region, NW India; Curr. Sci. 94 1582-1588.

Kumar R and Shrivastava J P 2009 Geochemistry of basic dykes from Betul-Jabalpur area in the Deccan volcanic province; J. Geol. Soc. India 74 95-107.

Le Bas M J, Le Maitre R W, Streckeisen A and Zanettin B 1986 IUGS subcommission on the systematics of igneous rocks: A chemical classification of volcanic rocks based on the total alkali-silica diagram; J. Petrol. 27(3) 745-750.

Lightfoot P and Hawkesworth C 1988 Origin of Deccan Trap lavas: Evidence from combined trace element and $\mathrm{Sr}-, \mathrm{Nd}-$ and Pb-isotope studies; Earth Planet. Sci. Lett. 91(1-2) 89-104.

Lightfoot P C, Hawkesworth C J, Devey C W, Rogers N W and Calsteren P V 1990 Source and differentiation of Deccan trap lavas: Implications of geochemical and mineral chemical variations; J. Petrol. 31(5) 1165-1200.

Mahoney J J, Duncan R A, Khan W, Gnos E and McCormick G R 2002 Cretaceous volcanic rocks of the South Tethyan suture zone, Pakistan: Implications for the Réunion hotspot and Deccan Traps; Earth Planet. Sci. Lett. 203 295-310.

McDonough W F 1990 Constraints on the composition of the continental lithospheric mantle; Earth Planet Sci. Lett. 101(1) 1-18.

McDonough W F and Sun S S 1995 The composition of the Earth; Chem. Geol. 120 223-253.

Melluso L, Barbieri M and Beccaluva L 2004 Chemical evolution, petrogenesis, and regional chemical correlations of the flood basalt sequence in the central Deccan Traps, India; J. Earth Syst. Sci. 113(4) 587-603.

Melluso L, Mahoney J J and Dallai L 2006 Mantle sources and crustal input as recorded in high-Mg Deccan Traps basalts of Gujarat (India); Lithos 89(3) 259-274.

Michael P J and Cheadle M J 2009 Making a crust; Science 323(5917) 1017-1018.

Nicholson H, Condomines M, Fitton J G, Fallick Ae, Grönvold K and Rogers G 1991 Geochemical and isotopic 
evidence for crustal assimilation beneath krafla, Iceland; J. Petrol. 32(5) 1005-1020.

Paul D, White W M and Blichert-Toft J 2005 Geochemistry of Mauritius and the origin of rejuvenescent volcanism on oceanic island volcanoes; Geochem. Geophys. Geosys. 6(6).

Pearce J A 2008 Geochemical fingerprinting of oceanic basalts with applications to ophiolite classification and the search for Archean oceanic crust; Lithos 100(1) 14-48.

Peng Z X, Mahoney J, Hooper P, Harris C and Beane J 1994 A role for lower continental crust in flood basalt genesis? Isotopic and incompatible element study of the lower six formations of the western Deccan Traps; Geochim. Cosmochim. Acta 58(1) 267-288.

Plank T and Langmuir C H 1998 The chemical composition of subducting sediment and its consequences for the crust and mantle; Chem. Geol. 145(3) 325-394.

Powell R 1984 Inversion of the assimilation and fractional crystallization (AFC) equations: Characterization of contaminants from isotope and trace element relationships in volcanic suites; J. Geol. Soc. London 141(3) 447-452.

Randive K 2015 Occurrence of unusual quartz xenocrystladen dykes in the Chhota Udaipur alkaline-carbonatite sub-province, Deccan Igneous Province, India; Curr. Sci. 108(12) 2261-2266.

Randive K R, Kumar J V, Korakoppa M M and Sahu M K 2017 Occurrence of REE mineralization in the layered Gabbros of Phenai Mata Igneous Complex, Gujarat, India; Curr. Sci. 112(2) 231-235.

Rao N V C, Burgess R, Lehmann B, Mainkar D, Pande S K, Hari K R and Bodhankar N $2011{ }^{40} \mathrm{Ar} /{ }^{39}$ Ar ages of mafic dykes from the Mesoproterozoic Chhattisgarh basin, Bastar craton, Central India: Implication for the origin and spatial extent of the Deccan Large Igneous Province; Lithos 125(3) 994-1005.

Ravi Kumar M and Mohan G 2005 Mantle discontinuities beneath the Deccan volcanic province; Earth Planet. Sci. Lett. 237(1) 252-263.

Ray J S 1998 Trace element and isotope evolution during concurrent assimilation, fractional crystallization, and liquid immiscibility of a carbonated silicate magma; Geochim. Cosmochim. Acta 62(19) 3301-3306.

Ray R, Sheth H C and Mallik J 2007 Structure and emplacement of the Nandurbar-Dhule mafic dyke swarm, Deccan Traps, and the tectonomagmatic evolution of flood basalts; Bull. Volcanol. 69(5) 537.

Rock N M S, Gwalani L G and Griffin B J 1994 Alkaline rocks and carbonatites of Amba Dongar and adjacent areas, Deccan Alkaline Province, Gujarat, India. 2. Complexly zoned clinopyroxene phenocrysts; Mineral. Petrol. 51(2) $113-135$

Rudnick R L and Gao S 2013 Composition of the continental crust; Treatise on Geochemistry, 659p.

Rudnick R L, McDonough W F, McCulloch M T and Taylor S R 1986 Lower crustal xenoliths from Queensland, Australia: Evidence for deep crustal assimilation and fractionation of continental basalts; Geochim. Cosmochim. Acta 50(6) 1099-1115.
Sen G 2001 Generation of Deccan trap magmas; Proc. Indian Acad. Sci. 110(4) 409-432.

Sen G, Bizimis M, Das R, Paul D K, Ray A and Biswas S 2009 Deccan plume, lithosphere rifting, and volcanism in Kutch, India; Earth Planet. Sci. Lett. 277 101-111.

Sheth H C and Melluso L 2008 The Mount Pavagadh volcanic suite, Deccan Traps: Geochemical stratigraphy and magmatic evolution; J. Asian Earth Sci. 32 5-21.

Sheth H C and Ray J S $2002 \mathrm{Rb} / \mathrm{Sr}-{ }^{87} \mathrm{Sr} /{ }^{86} \mathrm{Sr}$ variations in Bombay trachytes and rhyolites (Deccan Traps): $\mathrm{Rb}-\mathrm{Sr}$ isochron, or AFC process?; Int. Geol. Rev. 44 624-638.

Sheth H C, Mahoney J J and Chandrasekharam D 2004 Geochemical stratigraphy of Deccan flood basalts of the Bijasan Ghat section, Satpura Range, India; J. Asian Earth Sci. 23(1) 127-139.

Shrivastava J P and Pattanayak S K 2002 Basalts of the eastern Deccan volcanic province, India; Gondwana Res. 5(3) 649-665.

Silver P G, Behn M D, Kelley K, Schmitz M and Savage B 2006 Understanding cratonic flood basalts; Earth Planet. Sci. Lett. 245 190-201.

Simonetti A, Goldstein S L, Schmidberger S S and Viladkar S G 1998 Geochemical and $\mathrm{Nd}, \mathrm{Pb}$, and $\mathrm{Sr}$ isotope data from Deccan alkaline complexes-inferences for mantle sources and plume-lithosphere interaction; J. Petrol. 39(11-12) 1847-1864.

Sun S S and McDonough W F 1989 Chemical and isotopic systematics of oceanic basalts: Implications for mantle composition and processes; J. Geol. Soc. London Spec. Publ. 42(1) 313-345.

Taylor S R and McLennan S M 1985 The continental crust: Its composition and evolution; An Examination of the Geochemical Record Preserved in Sedimentary Rocks.

Tegner C, Wilson J R and Robins B 2005 Crustal assimilation in basalt and jotunite: Constraints from layered intrusions; Lithos 83(3) 299-316.

Vijaya Kumar K, Chavan C, Sawant S, Raju K N, Kanakdande P, Patode S, Deshpande K, Krishnamacharyulu S K, Vaideswaran $\mathrm{T}$ and Balaram V 2010 Geochemical investigation of a semi-continuous extrusive basaltic section from the Deccan Volcanic Province, India: Implications for the mantle and magma chamber processes; Contrib. Mineral. Petrol. 159(6) 839-862.

Wilson M 1989 Review of igneous petrogenesis: A global tectonic approach; Terra Nova 1 218-222.

Wood D A 1980 The application of a Th-Hf-Ta diagram to problems of tectonomagmatic classification and to establishing the nature of crustal contamination of basaltic lavas of the British Tertiary Volcanic Province; Earth Planet. Sci. Lett. 50 11-30.

Xiao L, Xu Y G, Mei H J, Zheng Y F, He B and Pirajno F 2004 Distinct mantle sources of low-Ti and high-Ti basalts from the western Emeishan large igneous province, SW China: Implications for plume-lithosphere interaction; Earth Planet. Sci. Lett. 228(3) 525-546. 